\title{
Face numbers and nongeneric initial ideals
}

\author{
Eric Babson and Isabella Novik \\ Department of Mathematics \\ University of Washington, Seattle, WA 98195-4350, USA \\ [babson, novik] @math. washington.edu
}

Submitted: Jun 30, 2005; Accepted: Dec 26, 2005; Published: Jan 3, 2006 Mathematics

Subject Classifications: 52B05, 13F55, 05E25

Dedicated to Richard Stanley on the occasion of his 60th birthday.

\begin{abstract}
Certain necessary conditions on the face numbers and Betti numbers of simplicial complexes endowed with a proper action of a prime order cyclic group are established. A notion of colored algebraic shifting is defined and its properties are studied. As an application a new simple proof of the characterization of the flag face numbers of balanced Cohen-Macaulay complexes originally due to Stanley (necessity) and Björner, Frankl, and Stanley (sufficiency) is given. The necessity portion of their result is generalized to certain conditions on the face numbers and Betti numbers of balanced Buchsbaum complexes.
\end{abstract}

\section{Introduction}

In this paper we study the face numbers of two classes of simplicial complexes: complexes endowed with a group action and balanced complexes. We accomplish this by exploring the behavior of a special (only partially generic) initial ideal of the Stanley-Reisner ideal of a simplicial complex.

The face numbers are basic invariants of simplicial complexes and their study goes back to Kruskal [14] and Katona [12] who characterized the face numbers of all finite simplicial complexes. Since then many powerful tools and techniques have been developed, among them are the theory of Stanley-Reisner rings and the method of algebraic shifting introduced by Kalai and closely related to the notion of generic initial ideals. Both techniques have resulted in many beautiful applications including the characterization of the face numbers of all Cohen-Macaulay complexes (due to Stanley [20]), the characterization of the flag face numbers of all balanced Cohen-Macaulay complexes (due to Stanley [21] (necessity) and Björner, Frankl, and Stanley [5] (sufficiency)), and the characterization of the face numbers of all simplicial complexes with prescribed Betti numbers (due to Björner and Kalai [6]). 
In the first part of this paper we prove certain necessary conditions on the face numbers and Betti numbers of simplicial complexes endowed with a group action. Our result is similar in spirit to the necessity portion of the Björner-Kalai theorem. In the second part we develop a version of algebraic shifting suitable for balanced simplicial complexes. We then utilize this technique to provide a new simpler proof of the characterization of the flag face numbers of balanced Cohen-Macaulay complexes, and to generalize the necessity portion of this result to get conditions on the face numbers and Betti numbers of balanced Buchsbaum complexes (e.g., simplicial manifolds).

We approach both problems by studying the combinatorics of a special (only partially generic) initial ideal of the Stanley-Reisner ideal of a simplicial complex. This method was first used in [17] for Buchsbaum complexes with symmetry; it is motivated by the original symmetric algebraic shifting due to Kalai [11] and Stanley's approach of exploiting special systems of parameters when the simplicial complex at hand has additional structure (see $[21,22,23])$.

We start by describing basic concepts and main results, deferring most of the definitions until the following sections.

A multicomplex $M$ on variables $x_{1}, \ldots, x_{n}$ is a collection of monomials in those variables that is closed under divisibility (i.e., $\mu^{\prime} \mid \mu \in M \Longrightarrow \mu^{\prime} \in M$ ). In contrast with the usual convention we do not require that each singleton $x_{i}, 1 \leq i \leq n$, be an element of $M$. The $F$-vector of $M$ is the vector $F(M)=\left(F_{0}, F_{1}, \ldots\right)$, where $F_{i}=F_{i}(M)$ denotes the number of monomials in $M$ of degree $i$. (Thus $F_{1} \leq n$ and $F_{0}=1$ unless $M$ is empty in which case $F_{0}=0$.)

A multicomplex $\Gamma$ is called a simplicial complex if all its elements are squarefree monomials. The elements of a simplicial complex $\Gamma$ are called faces, and the maximal ones (under divisibility) are called facets. We say that $\mu \in \Gamma$ is an $i$-dimensional face (or an $i$-face) if $\operatorname{deg} \mu=i+1$. (0-faces are usually referred to as vertices.) We also define the dimension of $\Gamma, \operatorname{dim} \Gamma$, as the maximal dimension of its faces. The $f$-vector of a $(d-1)$ dimensional simplicial complex $\Gamma$ is the vector $f(\Gamma)=\left(f_{-1}, f_{0}, f_{1}, \ldots, f_{d-1}\right)$, where $f_{i}$ denotes the number of $i$-faces of $\Gamma$. Thus for a simplicial complex $\Gamma, f(\Gamma)$ differs from $F(\Gamma)$ only by a shift in the indexing.

Denote by $\widetilde{H}_{i}(\Gamma, \mathbf{k})$ the $i$ th reduced simplicial homology of $\Gamma$ with coefficients in $\mathbf{k}$, by $\beta_{i}(\Gamma)=\operatorname{dim}_{\mathbf{k}} \widetilde{H}_{i}(\Gamma ; \mathbf{k})$ the $i$ th reduced Betti number of $\Gamma$, and by $\chi_{i}(\Gamma)=\operatorname{rk} \partial_{i+1}$ the rank of the $i$ th differential $\partial_{i+1}: C_{i+1}(\Gamma) \rightarrow C_{i}(\Gamma)$ in the reduced simplicial chain complex for $\Gamma$. In particular $f_{i}=\beta_{i}+\chi_{i}+\chi_{i-1}$, and $\chi_{-1}=f_{-1}-\beta_{-1}=1$ unless $\operatorname{dim} \Gamma=-1$ in which case $\chi_{-1}=0$. The sequence $\left\{\beta_{i}(\Gamma)\right\}_{i=-1}^{\operatorname{dim} \Gamma}$ is called the Betti sequence of $\Gamma$ (over $\mathbf{k}$ ).

Our first result provides certain necessary conditions on the $f$-vector and the Betti sequence of a simplicial complex endowed with a proper group action. The general statement is given in Section 3. In the case of a centrally symmetric complex (that is, a complex admitting a free action of $\mathbb{Z} / 2 \mathbb{Z}$ ) our result reduces to the following theorem.

Theorem 1.1 If $\Gamma$ is a subcomplex of the $m$-dimensional cross polytope and $1 \leq k \leq$ $\operatorname{dim} \Gamma$ then there exists a multicomplex $M_{k}$ on $2 m-k$ variables such that

1. all elements of $M_{k}$ are squarefree in the first $m$ variables; 


\section{2. $F_{k}\left(M_{k}\right)=\chi_{k-1}(\Gamma)$ and $F_{k+1}\left(M_{k}\right)=f_{k}(\Gamma)$.}

For comparison recall that the theorem of Björner-Kalai [6] asserts that two sequences of nonnegative integers $\left(1, f_{0}, f_{1}, \ldots, f_{d-1}\right)$ and $\left(0, \beta_{0}, \beta_{1}, \ldots, \beta_{d-1}\right)$ with equal alternating sums form the $f$-vector and the Betti sequence of some $(d-1)$-dimensional $(d \geq 1)$ simplicial complex $\Gamma$ if and only if for every $1 \leq k \leq d-1$ there exists a squarefree multicomplex $\Delta_{k}$ such that $F_{k}\left(\Delta_{k}\right)=\chi_{k-1}(\Gamma)$ and $F_{k+1}\left(\Delta_{k}\right)=f_{k}(\Gamma)-\chi_{k-1}(\Gamma)$. We remark that $\Delta_{k}$ can be easily reconstructed from a multicomplex $M_{k}$ in the statement of Theorem 1.1 (see also Theorem 3.2).

A numerical relationship between the number of $(k-1)$-faces and the number of $k$-faces in a simplicial complex is given by Kruskal-Katona theorem [14, 12], and the relationship between the number of monomials of degree $k$ and those of degree $k+1$ in a multicomplex is provided by Macaulay's theorem [15]. Clements and Lindström [7] generalized both results by finding explicit inequalities relating the number of monomials of degree $k$ to those of degree $k+1$ in a multicomplex with specified upper bounds on degrees of some of the variables (such as for example a multicomplex $M_{k}$ in the statement of Theorem 1.1).

Thus by the Clements-Lindström theorem verification of the combinatorial conditions of Theorem 1.1 reduces to verification of a certain system of inequalities. While Theorem 1.1 is sharp in the sense that if $\Gamma$ is a skeleton of (the boundary complex of) the $m$ dimensional cross polytope, then all those inequalities hold as equalities (see Remark 3.4 ), its conditions are probably not sufficient conditions on the $f$-numbers and Betti numbers of centrally symmetric complexes.

The second part of the paper deals with colored multicomplexes and balanced simplicial complexes introduced in [21]. To this end, we assume that the set of variables $V$ is endowed with an ordered partition $\left(V_{1}, \ldots, V_{r}\right)$. A multicomplex $M$ on $V$ is called a-colored, where $\mathbf{a}=\left(a_{1}, \ldots, a_{r}\right) \in \mathbb{Z}_{+}^{r}$ is a fixed sequence of positive integers, if for every $1 \leq i \leq r$ no element of $M$ that involves only variables from $V_{i}$ has degree $>a_{i}$. We say that a $(d-1)$-dimensional simplicial complex $\Gamma$ is a-balanced if it is a-colored and $\sum_{i=1}^{r} a_{i}=d$. Thus, $(1,1, \ldots, 1)$-colored multicomplexes are simplicial complexes, and a simplicial complex is a-balanced for $\mathbf{a} \in \mathbb{Z}^{1}$ if and only if it is (a-1)-dimensional.

In this paper we develop a notion of colored algebraic shifting — an algebraic operation that associates with a colored simplicial complex $\Gamma$ another colored simplicial complex, $\tilde{\Delta}(\Gamma)$. This new complex is color-shifted (as defined in section 5), has the same flag $f$-vector as $\Gamma$, and is Cohen-Macaulay if $\Gamma$ is a-balanced and Cohen-Macaulay.

Stanley's celebrated theorem [20], [24, Thm. II.3.3] characterized the $f$-vectors of all Cohen-Macaulay (CM for short) simplicial complexes. It was then generalized by Stanley [21] and Björner, Frankl, and Stanley [5] to a complete (combinatorial) characterization of the flag $f$-vectors of a-balanced CM complexes (see Theorem 6.3). In the language of the ordinary face numbers their result reduces to the assertion that a sequence $h=$ $\left(h_{0}, h_{1}, \ldots, h_{d}\right) \in \mathbb{Z}^{d+1}$ is the $h$-vector of an a-balanced CM complex if and only if $h$ is the $F$-vector of an a-colored multicomplex, where the $h$-vector of a $(d-1)$-dimensional simplicial complex $\Gamma$ is the vector $h(\Gamma)=\left(h_{0}(\Gamma), h_{1}(\Gamma), \ldots, h_{d}(\Gamma)\right)$ whose entries satisfy 
the following relation

$$
\sum_{i=0}^{d} h_{i}(\Gamma) x^{d-i}=\sum_{i=0}^{d} f_{i-1}(\Gamma)(x-1)^{d-i}
$$

Here we use colored algebraic shifting to provide a simple proof of the Stanley-BjörnerFrankl theorem. (The original proof of the sufficiency part relied on the notion of combinatorial shifting.) We also generalize the necessity portion of that result to certain conditions on the $f$-vector and Betti sequence of an a-balanced Buchsbaum complex (Theorem 6.6).

The structure of this paper is as follows. In Section 2 we review basic facts on StanleyReisner rings and initial ideals, and then introduce and study certain monomial sets that are at the root of all our proofs. In Section 3 after recalling some notions related to group actions, we apply the results of Section 2 to complexes with symmetry. The proof of Theorem 1.1 is completed in Section 4. Section 5 is devoted to developing the notion of colored algebraic shifting and studying its properties. Section 6 contains a new proof of the Stanley-Björner-Frankl theorem as well as the proof of Theorem 6.6 on a-balanced Buchsbaum complexes.

\section{The Stanley-Reisner ring, initial ideals, and mono- mial sets}

Let $\mathbf{k}$ be an arbitrary infinite field. Consider the polynomial ring $\mathbf{k}[\mathbf{x}]:=\mathbf{k}\left[x_{1}, \ldots, x_{n}\right]$ with the grading $\operatorname{deg} x_{i}=1$ for all $1 \leq i \leq n$. Let $\mathbb{N}$ denote the set of non-negative integers. Identifying a function $f:[n] \rightarrow \mathbb{N}$ in $\mathbb{N}^{[n]}$ (here $[n]=[1, n]=\{1, \ldots, n\}$ ) with the monomial $\prod_{i \in[n]} x_{i}^{f(i)}$, denote by $\mathbb{N}^{[n]}$ the set of all monomials of $\mathbf{k}[\mathbf{x}]$, and consider $\mathbb{N}^{[n]}$ as a multiplicative monoid. Thus $\{0,1\}^{[n]}$ is the set of squarefree monomials. For $\sigma \subseteq[n]$ we let $\mathbb{N}^{\sigma}$ denote the set of all monomials in the variables $x_{i}$ with $i \in \sigma$ (e.g. $\mathbb{N}^{\emptyset}=\{1\}$ ), and $\mathbb{N}_{r}^{\sigma}$ denote the set of elements of degree $r$ in $\mathbb{N}^{\sigma}$.

If $\Gamma \subseteq\{0,1\}^{[n]}$ is a simplicial complex then the Stanley-Reisner ideal of $\Gamma[24$, Def. II.1.1] is the squarefree monomial ideal

$$
I_{\Gamma}:=\left\langle\{0,1\}^{[n]}-\Gamma\right\rangle \subset \mathbf{k}[\mathbf{x}] .
$$

The ring $\mathbf{k}[\mathbf{x}] / I_{\Gamma}$ is called the Stanley-Reisner ring (or the face ring) of $\Gamma$.

We fix the reverse lexicographic order $\succ$ on the set of all monomials of $\mathbf{k}[\mathbf{x}]$ that refines the partial order by degree and satisfies $x_{1} \succ x_{2} \succ \ldots \succ x_{n}$ (e.g. $x_{1}^{2} \succ x_{1} x_{2} \succ$ $\left.x_{2}^{2} \succ x_{1} x_{3} \succ x_{2} x_{3} \succ x_{3}^{2} \succ \cdots\right)$. Every $u \in G L_{n}(\mathbf{k})$ defines a graded automorphism of $\mathbf{k}[\mathbf{x}]$ via $u\left(x_{j}\right)=\sum_{i=1}^{n} u_{i j} x_{i}$. In particular, for a simplicial complex $\Gamma \subseteq\{0,1\}^{[n]}, u I_{\Gamma}$ is a homogeneous ideal of $\mathbf{k}[\mathbf{x}]$. Thus $\operatorname{in}\left(u I_{\Gamma}\right)$ 一 the reverse lexicographic initial ideal of $u I_{\Gamma}$ is a well-defined monomial ideal [8, Section 15.2], and hence the collection of monomials

$$
B_{u, \Gamma}:=\mathbb{N}^{[n]}-\operatorname{in}\left(u I_{\Gamma}\right)
$$


is a multicomplex.

The central idea of this paper is that for a suitably chosen $u$ one can read off the $f$-numbers and the Betti numbers of $\Gamma$ from the set $B_{u, \Gamma}$ (see Lemma 2.2 below). The multicomplexes appearing in the statements of Theorems 1.1 and 6.6 then can be realized as subcomplexes of $B_{u, \Gamma}$. In the case of a generic $u$ this idea is originally due to Kalai [11]. The main novelty of our approach, a development of which was started in [17], is that $u$ need not be completely generic.

To state Lemma 2.2 we need to review several additional facts and definitions. We start by remarking that the only property of reverse lexicographic order we use in this paper is [8, Prop. 15.12], asserting that for every homogeneous ideal $I \subseteq \mathbf{k}[\mathbf{x}]$,

$$
\operatorname{in}\left(I+\left\langle x_{n}\right\rangle\right)=\operatorname{in}(I)+\left\langle x_{n}\right\rangle \quad \text { and } \operatorname{in}\left(I: x_{n}\right)=\left(\operatorname{in}(I): x_{n}\right),
$$

where the ideal $\left(I: x_{n}\right)$ is defined as $\left\{\nu \in \mathbf{k}[\mathbf{x}] \mid \nu x_{n} \in I\right\}$. This readily leads to

$$
\begin{aligned}
& \operatorname{in}\left(I+\left\langle x_{n-k+1}, \ldots, x_{n}\right\rangle\right)=\operatorname{in}(I)+\left\langle x_{n-k+1}, \ldots, x_{n}\right\rangle \quad \forall 0 \leq k \leq n \text { and } \\
& \operatorname{in}\left(\left(I+\left\langle x_{n-k+1}, \ldots, x_{n}\right\rangle\right): x_{n-k}\right)=\left(\left(\operatorname{in}(I)+\left\langle x_{n-k+1}, \ldots, x_{n}\right\rangle\right): x_{n-k}\right) .
\end{aligned}
$$

For a simplicial complex $\Gamma \subseteq\{0,1\}^{[n]}$ and a matrix $u \in G L_{n}(\mathbf{k})$, we consider the family

$$
J_{u, \Gamma}\langle k\rangle:=u I_{\Gamma}+\left\langle x_{n-k+1}, \ldots, x_{n}\right\rangle, \quad 0 \leq k \leq n,
$$

of graded ideals of $\mathbf{k}[\mathbf{x}]$, and the following two families of subsets of $B_{u, \Gamma}$ :

$$
B_{u, \Gamma}\langle k\rangle:=B_{u, \Gamma} \cap \mathbb{N}^{[n-k]} \quad \text { and } \quad Z_{u, \Gamma}\langle k\rangle:=\left\{\nu \in B_{u, \Gamma}\langle k\rangle: \nu x_{n-k} \notin B_{u, \Gamma}\right\},
$$

$0 \leq k \leq n-1$. We also write $B_{u, \Gamma}\langle k\rangle_{l}$ and $Z_{u, \Gamma}\langle k\rangle_{l}$ to denote the set of elements of degree $l$ in $B_{u, \Gamma}\langle k\rangle$ and $Z_{u, \Gamma}\langle k\rangle$, respectively. The following proposition summarizes some elementary properties of these monomial sets. (Note that $J_{u, \Gamma}\langle 0\rangle=u I_{\Gamma}, B_{u, \Gamma}\langle 0\rangle=B_{u, \Gamma}$, and that the definition of $B_{u, \Gamma}\langle k\rangle$ makes sense for $k<0$ as well, e.g. $B_{u, \Gamma}\langle-1\rangle=B_{u, \Gamma} \cap$ $\mathbb{N}^{[n+1]}=B_{u, \Gamma}$. We use the case of $k=-1$ as the base case for several inductive proofs below.)

Proposition 2.1 Let $\Gamma \subseteq \Lambda \subseteq\{0,1\}^{[n]}$ be simplicial complexes, and let $u \in G L_{n}(\mathbf{k})$. Then the following holds:

1. $B_{u, \Gamma} \subseteq B_{u, \Lambda}$.

2. For all $0 \leq k \leq n-1, B_{u, \Gamma}\langle k\rangle=\mathbb{N}^{[n]}-\operatorname{in}\left(J_{u, \Gamma}\langle k\rangle\right)$ and

$$
B_{u, \Gamma}\langle k\rangle-Z_{u, \Gamma}\langle k\rangle=\mathbb{N}^{[n]}-\operatorname{in}\left(J_{u, \Gamma}\langle k\rangle: x_{n-k}\right) .
$$

Thus, the sets $B_{u, \Gamma}\langle k\rangle$ and $B_{u, \Gamma}\langle k\rangle-Z_{u, \Gamma}\langle k\rangle$ are multicomplexes that provide $\mathbf{k}$-bases for $\mathbf{k}[\mathbf{x}] / J_{u, \Gamma}\langle k\rangle$ and $\mathbf{k}[\mathbf{x}] /\left(J_{u, \Gamma}\langle k\rangle: x_{n-k}\right)$, respectively. 
3. The generating function of $B=B_{u, \Gamma}, P(B, t):=\sum_{k \geq 0}\left|B_{k}\right| t^{k}$, equals

$$
\sum_{k=0}^{\operatorname{dim} \Gamma+1} \frac{f_{k-1}(\Gamma) t^{k}}{(1-t)^{k}}
$$

Proof: Since $\Gamma \subseteq \Lambda$, it follows that $I_{\Gamma} \supseteq I_{\Lambda}$, and hence that

$$
B_{u, \Gamma}=\mathbb{N}^{[n]}-\operatorname{in}\left(u I_{\Gamma}\right) \subseteq \mathbb{N}^{[n]}-\operatorname{in}\left(u I_{\Lambda}\right)=B_{u, \Lambda},
$$

implying part 1. Part 2 is a consequence of equations (1) and (2), and [8, Thm. 15.3]. Finally, since $B_{u, \Gamma}$ is a $\mathbf{k}$-basis of $\mathbf{k}[\mathbf{x}] / u I_{\Gamma}$, and since $\mathbf{k}[\mathbf{x}] / u I_{\Gamma}$ is a graded algebra over $\mathbf{k}$ isomorphic to $\mathbf{k}[\mathbf{x}] / I_{\Gamma}, P(B, t)$ coincides with the Hilbert series of $\mathbf{k}[\mathbf{x}] / I_{\Gamma}$. Theorem II.1.4 of [24] then yields part 3 .

Assume now that $\Gamma \subseteq\{0,1\}^{[n]}$ is a $(d-1)$-dimensional simplicial complex. Since $\mathbf{k}[\mathbf{x}] / u I_{\Gamma}$ is isomorphic to $\mathbf{k}[\mathbf{x}] / I_{\Gamma}$, we infer from [24, Thm. II.1.3] that the Krull dimension of $\mathbf{k}[\mathbf{x}] / u I_{\Gamma}$ (i.e., the maximum number of algebraically independent elements over $\mathbf{k}$ in $\left.\mathbf{k}[\mathbf{x}] / u I_{\Gamma}\right)$ is $d$. In fact, by the result due to Kind and Kleinschmidt [13], [24, Lemma III.2.4(a)], the $d$ elements $x_{n-d+1}, \ldots, x_{n}$ form a linear system of parameters, abbreviated l.s.o.p., for $\mathbf{k}[\mathbf{x}] / u I_{\Gamma}$ (the condition that implies being algebraically independent over $\mathbf{k}$ ) if and only if $u \in G L_{n}(\mathbf{k})$ possesses the following property referred to as the KindKleinschmidt condition:

- for every face $x_{i_{1}} \cdots x_{i_{k}} \in \Gamma$, the submatrix of $u^{-1}$ defined by the intersection of its last $d$ columns and the rows numbered $i_{1}, \ldots, i_{k}$ has rank $k$.

We say that $u$ satisfies the strong Kind-Kleinschmidt condition with respect to $\Gamma$ if

- for every face $x_{i_{1}} \cdots x_{i_{k}} \in \Gamma$, the submatrix of $u^{-1}$ defined by the intersection of its last $k$ columns and the rows numbered $i_{1}, \ldots, i_{k}$ is nonsingular.

Thus if $u$ satisfies the strong Kind-Kleinschmidt condition with respect to $\Gamma$ (there is at least one such $u$ if $\mathbf{k}$ is infinite), then it satisfies this condition w.r.t. any subcomplex of $\Gamma$. In particular, $x_{n-k+1}, \ldots, x_{n}$ is an l.s.o.p. for $\mathbf{k}[\mathbf{x}] / u I_{\Sigma}$ for every $0 \leq k \leq \operatorname{dim} \Gamma+1$ and every $(k-1)$-dimensional subcomplex $\Sigma \subseteq \Gamma$. Therefore, for such $u$ and $\Sigma$, all homogeneous components of $\mathbf{k}[\mathbf{x}] / J_{u, \Sigma}\langle k\rangle$ starting from the $(k+1)$-th component and up vanish (see [24, Lemma III.2.4(b)]), and we conclude from Proposition 2.1(2) that

$$
B_{u, \Sigma}\langle k\rangle_{k+1}=\emptyset
$$

which will be of use later.

We now come to the main tool of this paper.

Lemma 2.2 Let $\Gamma \subseteq\{0,1\}^{[n]}$ be a simplicial complex and let $u \in G L_{n}(\mathbf{k})$ be a matrix satisfying the strong Kind-Kleinschmidt condition with respect to $\Gamma$. Then the monomial sets $B_{u, \Gamma}\langle k\rangle$ and $Z_{u, \Gamma}\langle k\rangle$ have the following properties: 
1. $\mu \mathbb{N}^{[n-k+1, n]} \subseteq B_{u, \Gamma}$ for all $\mu \in B_{u, \Gamma}\langle k-1\rangle_{k}$ and all $0 \leq k \leq n$.

2. $\left|B_{u, \Gamma}\langle k-1\rangle_{k}\right|=f_{k-1}(\Gamma)$ for all $0 \leq k \leq n$.

3. $\left|Z_{u, \Gamma}\langle k\rangle_{k}\right|=\beta_{k-1}(\Gamma)$ for all $0 \leq k \leq n-1$ and $Z_{u, \Gamma}\langle k\rangle_{l}=\emptyset$ for all $l \geq k+1$.

If $u \in G L_{n}(\mathbf{k})$ is generic then it satisfies the strong Kind-Kleinschmidt condition with respect to any simplicial complex $\Gamma \subseteq\{0,1\}^{[n]}$. In this special case (with the additional restriction that $\mathbf{k}$ is a field of characteristic zero) Lemma 2.2 is not new: its parts 1 and 2 are [11, Lemma 6.3], and part 3 follows from Corollary 2.5 and Lemma 2.6 of [2].

In the rest of this section we discuss an application of Lemma 2.2 to the face numbers and Betti numbers of simplicial complexes deferring its somewhat technical proof until Section 4. Throughout this discussion we fix a simplicial complex $\Gamma$ and a matrix $u$ satisfying the strong Kind-Kleinschmidt condition w.r.t $\Gamma$, and write $B=B_{u, \Gamma}, Z=Z_{u, \Gamma}$, $f_{k}=f_{k}(\Gamma), \beta_{k}=\beta_{k}(\Gamma)$, and $\chi_{k}=\chi_{k}(\Gamma)$.

Lemma 2.3 $\left|B\langle k\rangle_{k}-Z\langle k\rangle_{k}\right|=\chi_{k-1}$ for every $0 \leq k \leq n-1$.

Proof: If $\mu \in B\langle k-1\rangle_{k}$, then either $\mu \in B\langle k\rangle_{k}$ or $x_{n-k+1} \mid \mu$. In the latter case, $\mu^{\prime}:=$ $\mu / x_{n-k+1}$ is an element of $B\langle k-1\rangle_{k-1}$ (since $B\langle k-1\rangle_{k-1}$ is a multicomplex), but is not an element of $Z\langle k-1\rangle_{k-1}$ (by definition of $Z\langle k-1\rangle$ ). Thus

$$
B\langle k-1\rangle_{k}=B\langle k\rangle_{k} \bigcup x_{n-k+1} \cdot\left(B\langle k-1\rangle_{k-1}-Z\langle k-1\rangle_{k-1}\right) .
$$

Parts 2 and 3 of Lemma 2.2 then imply that

$$
\begin{aligned}
\left|B\langle k\rangle_{k}-Z\langle k\rangle_{k}\right| & =\left|B\langle k-1\rangle_{k}\right|-\left|Z\langle k\rangle_{k}\right|-\left|B\langle k-1\rangle_{k-1}-Z\langle k-1\rangle_{k-1}\right| \\
& =\left(f_{k-1}-\beta_{k-1}\right)-\left|B\langle k-1\rangle_{k-1}-Z\langle k-1\rangle_{k-1}\right|,
\end{aligned}
$$

and the assertion follows by induction on $k$. For the $k=0$ case note that

$$
B\langle 0\rangle_{0}=B \cap N_{0}^{[n]}=B \cap\{1\}=\left\{\begin{array}{cc}
\{1\} & \text { if } u I_{\Gamma} \neq\langle 1\rangle \\
\emptyset & \text { if } u I_{\Gamma}=\langle 1\rangle
\end{array}=\left\{\begin{array}{cc}
\{1\} & \text { if } \Gamma \neq \emptyset \\
\emptyset & \text { if } \Gamma=\emptyset
\end{array}\right.\right.
$$

and so $\left|B\langle 0\rangle_{0}\right|=f_{-1}$, which together with $\left|Z\langle 0\rangle_{0}\right|=\beta_{-1}$ implies the assertion.

Proposition 2.1 and Lemmas 2.2 and 2.3 yield the following result.

Theorem 2.4 Let $\Lambda \subseteq\{0,1\}^{[n]}$ be a simplicial complex, let $u \in G L_{n}(\mathbf{k})$ be a matrix satisfying the strong Kind-Kleinschmidt condition w.r.t. $\Lambda$, and let $\Gamma$ be a subcomplex of $\Lambda$. Then for every $0 \leq k \leq \operatorname{dim} \Gamma$, there exists a multicomplex $M_{k} \subseteq B_{u, \Lambda}\langle k\rangle$ such that $F_{k}\left(M_{k}\right)=\chi_{k-1}(\Gamma)$ and $F_{k+1}\left(M_{k}\right)=f_{k}(\Gamma)$.

Proof: Define $M_{k}=B_{u, \Gamma}\langle k\rangle-Z_{u, \Gamma}\langle k\rangle . \quad M_{k}$ is a multicomplex by Proposition 2.1(2), $F_{k+1}\left(M_{k}\right)=f_{k}(\Gamma)$ by Lemma $2.2(2,3)$, and $F_{k}\left(M_{k}\right)=\chi_{k-1}(\Gamma)$ by Lemma 2.3. Also since $\Gamma \subseteq \Lambda$, Proposition 2.1(1) yields that $M_{k} \subseteq B_{u, \Gamma} \subseteq B_{u, \Lambda}$. 


\section{Complexes with a group action}

The goal of this section is to deduce Theorem 1.1 along with its generalization for complexes with a proper action of a cyclic group of prime order from Theorem 2.4. We start by setting up the notation and reviewing basic facts and definitions related to complexes with a group action. Our exposition follows [17]. Throughout this section let $\Gamma \subseteq\{0,1\}^{[n]}$ be a simplicial complex on the vertex set $\left\{x_{1}, \ldots, x_{n}\right\}$, and let $G=\mathbb{Z} / p \mathbb{Z}$ be a cyclic group of prime order.

A bijection $\sigma:[n] \rightarrow[n]$ defines a natural map $\sigma:\{0,1\}^{[n]} \rightarrow\{0,1\}^{[n]}$. This map is called a (simplicial) automorphism of $\Gamma$ if for every face $\mathcal{F} \in \Gamma, \sigma(\mathcal{F}) \in \Gamma$ as well. Denote by $\operatorname{Aut}(\Gamma)$ the group of all automorphisms of $\Gamma$. An action of group $G$ on $\Gamma$ is a homomorphism $\pi: G \rightarrow \operatorname{Aut}(\Gamma)$. An action $\pi$ of $G$ is proper if

$$
\pi(h)(\mathcal{F})=\mathcal{F} \text { for some } h \in G, \mathcal{F}=x_{i_{1}} \ldots x_{i_{k}} \in \Gamma \Longrightarrow \pi(h)\left(x_{i_{j}}\right)=x_{i_{j}} \forall 1 \leq j \leq k,
$$

and is free if

$$
\pi(h)(\mathcal{F})=\mathcal{F} \text { for some } \mathcal{F} \in \Gamma, \mathcal{F} \neq 1 \Longrightarrow h \text { is the unit element of } G \text {. }
$$

\section{Example 3.1}

1. Let $\Delta^{p-1}$ be a $(p-1)$-dimensional simplex with all its faces and let $\partial \Delta^{p-1}$ be its boundary complex. Letting the generator of $G$ cyclically permute the $p$ vertices of the simplex defines a free $G$-action on $\partial \Delta^{p-1}$ (but a nonfree and nonproper action on $\Delta^{p-1}$.)

2. Recall that if $\Gamma_{1}$ and $\Gamma_{2}$ are simplicial complexes on two disjoint vertex sets $V_{1}$ and $V_{2}$, then their join $\Gamma_{1} * \Gamma_{2}:=\left\{\mu_{1} \cdot \mu_{2}: \mu_{1} \in \Gamma_{1}, \mu_{2} \in \Gamma_{2}\right\}$ is a simplicial complex on $V_{1} \cup V_{2}$. A pair of proper $G$-actions $\pi_{i}: G \rightarrow \operatorname{Aut}\left(\Gamma_{i}\right)(i=1,2)$ defines a proper action $\pi: G \rightarrow \operatorname{Aut}\left(\Gamma_{1} * \Gamma_{2}\right)$ via $\pi(h)\left(\mu_{1} \cdot \mu_{2}\right)=\pi_{1}(h)\left(\mu_{1}\right) \cdot \pi_{2}(h)\left(\mu_{2}\right)$.

Assume $\Gamma$ is endowed with a $G$-action $\pi$. For a vertex $v$ of $\Gamma$, define the $G$-orbit of $v$ as $\operatorname{Orb}(v):=\{\pi(h)(v): h \in G\}$. Since $|G|=p$ is a prime number, for a vertex $v$ of $\Gamma$, either $|\operatorname{Orb}(v)|=1$ (in which case $v$ is said to be $G$-invariant) or $|\operatorname{Orb}(v)|=p$ (we call such an orbit a free $G$-orbit). Thus if $l$ denotes the number of $G$-invariant vertices and $m$ the number of free $G$-orbits, then $n=l+p m$. To simplify notation we assume from now on that the last $l$ vertices $x_{p m+i}, 1 \leq i \leq l$, are $G$-invariant and that $\operatorname{Orb}\left(x_{i}\right)=\left\{x_{i+j m}: 0 \leq j \leq p-1\right\}$ for $1 \leq i \leq m$. Note that if the $G$-action on $\Gamma$ is proper then no free $G$-orbit forms a face of $\Gamma$, and hence

$$
x_{i} x_{i+m} \cdots x_{i+(p-1) m} \notin \Gamma \quad \text { for all } 1 \leq i \leq m .
$$

For arbitrary integers $p \geq 2, m, l \geq 0$ (with $p$ prime or composite), we define $\Lambda(p, m, l)$ to be the maximal subcomplex of $\{0,1\}^{[p m+l]}$ satisfying Eq. (4). It is straightforward to see that

$$
\Lambda(p, m, l):=\partial \Delta_{1}^{p-1} * \ldots * \partial \Delta_{m}^{p-1} * \Delta^{l-1}
$$


where $\partial \Delta_{i}^{p-1}(i=1, \ldots, m)$ is the boundary complex of the $(p-1)$-dimensional simplex on the vertex set $\left\{x_{i+j m}: 0 \leq j \leq p-1\right\}$, and $\Delta^{l-1}$ is the simplex (with all its faces) on the vertex set $\left\{x_{p m+j}: 1 \leq j \leq l\right\}$. In particular, $\Lambda(2, m, 0)$ is the boundary of the $m$-dimensional cross polytope $C_{m}^{\Delta}$. If $p$ is a prime, let $G=\mathbb{Z} / p \mathbb{Z}$ act freely on $\partial \Delta_{i}^{p-1}(1 \leq i \leq m)$, and trivially on $\Delta^{l-1}$. This defines a proper $G$-action on $\Lambda(p, m, l)$. Moreover, $\Lambda(p, m, l)$ is the maximal subcomplex of $\{0,1\}^{[p m+l]}$ among all the complexes that are endowed with a proper $G$-action and have $m$ free $G$-orbits and $l G$-invariant vertices.

Recall that in our notation, $[0, p-1]^{[m]} \times \mathbb{N}^{[m+1, n]}$ denotes the set of monomials $\left\{x_{1}^{a_{1}} x_{2}^{a_{2}} \cdots x_{n}^{a_{n}} \in \mathbb{N}^{[n]}: a_{i} \leq p-1 \forall i \in[m]\right\}$. We are now in a position to prove the following generalization of Theorem 1.1.

Theorem 3.2 If $\Gamma$ is a subcomplex of $\Lambda(p, m, l)$ (where $p \geq 2, m, l \geq 0$ are arbitrary integers), $n=p m+l$, and $1 \leq k \leq \operatorname{dim}(\Gamma)$ then there exists a multicomplex $M_{k} \subseteq$ $[0, p-1]^{[m]} \times \mathbb{N}^{[m+1, n-k]}$ such that $F_{k}\left(M_{k}\right)=\chi_{k-1}(\Gamma)$ and $F_{k+1}\left(M_{k}\right)=f_{k}(\Gamma)$.

Corollary 3.3 If $\Gamma \subseteq\{0,1\}^{[n]}$ is a simplicial complex that admits a proper action of $G=\mathbb{Z} / p \mathbb{Z}$ for a prime $p$ and has $m$ free $G$-orbits, and $1 \leq k \leq \operatorname{dim}(\Gamma)$, then there exists a multicomplex $M_{k} \subseteq[0, p-1]^{[m]} \times \mathbb{N}^{[m+1, n-k]}$ such that $F_{k}\left(M_{k}\right)=\chi_{k-1}(\Gamma)$ and $F_{k+1}\left(M_{k}\right)=f_{k}(\Gamma)$.

The importance of Corollary 3.3 is that (together with the Clements-Lindström theorem [7]) it imposes strong restrictions on the possible face numbers and Betti numbers of a simplicial complex with a proper $\mathbb{Z} / p \mathbb{Z}$-action.

Proof of Theorem 3.2: By Theorem 2.4, to prove the statement it suffices to construct a matrix $u$ satisfying the strong Kind-Kleinschmidt condition w.r.t. $\Lambda:=\Lambda(p, m, l)$ and such that $B_{u, \Lambda} \subseteq[0, p-1]^{[m]} \times \mathbb{N}^{[m+1, n]}$. A construction of such a matrix was given in the proof of [17, Theorem 3.3]. For completeness we briefly outline it here. We replace field $\mathbf{k}$ by a larger field $\mathbf{K}=\mathbf{k}\left(y_{i j}, w_{i j}, z_{i j}\right)$ of rational functions in $(p-1)^{2} m^{2}+l^{2}+p m l$ variables and perform all computations inside $\mathbf{K}[\mathbf{x}]$ rather than $\mathbf{k}[\mathbf{x}]$. For instance, we regard $I_{\Lambda}$ and $B_{u, \Lambda}$ as an ideal and a subset of $\mathbf{K}[\mathbf{x}]$, respectively. Let $Y=\left(y_{i j}\right), W=\left(w_{i j}\right)$ and $Z=\left(z_{i j}\right)$ be $(p-1) m \times(p-1) m, l \times l$ and $p m \times l$ matrices respectively. Let $I_{m}$ denote the $m \times m$ identity matrix, let $E=\left[I_{m}\left|I_{m}\right| \cdots \mid I_{m}\right]$ be the $m \times(p-1) m$ matrix consisting of $(p-1)$ blocks of $I_{m}$, and let $O$ be the zero-matrix. Define

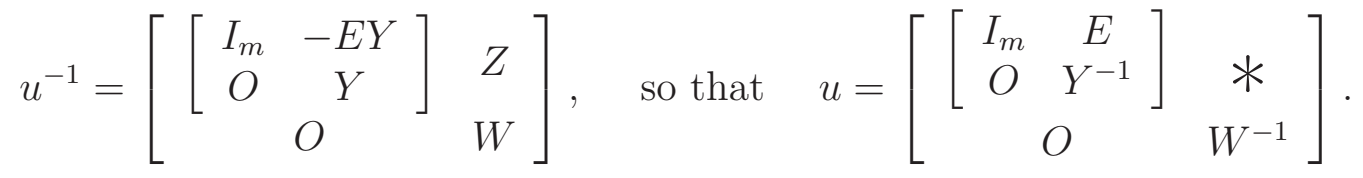

In particular $u_{s, i+j m}=0$ for all $1 \leq s<i \leq m$ and $0 \leq j \leq p-1$.

Since $x_{i} x_{i+m} \cdots x_{i+(p-1) m} \in I_{\Lambda}$ for $1 \leq i \leq m$, it follows that

$$
u I_{\Lambda} \ni \prod_{j=0}^{p-1} u\left(x_{i+j m}\right)=\prod_{j=0}^{p-1}\left(x_{i}+\sum_{s>i} u_{s, i+j m} x_{s}\right)=x_{i}^{p}+\sum\left\{\alpha_{\mu} \mu: x_{i}^{p} \succ \mu\right\} .
$$


Thus $x_{i}^{p} \in \operatorname{in}\left(u I_{\Lambda}\right), 1 \leq i \leq m$, implying that $B_{u, \Lambda} \subseteq[0, p-1]^{[m]} \times \mathbb{N}^{[m+1, n]}$.

The fact that $u$ satisfies the strong Kind-Kleinschmidt condition w.r.t. $\Lambda$ follows easily from the definitions of $u$ and $\Lambda$ (see the proof of [17, Thm. 3.3]).

Remark 3.4 The assertion of Theorem 3.2 is the best possible in the following sense. If $\Gamma$ is the $s$-dimensional skeleton of $\Lambda(p, m, l)$ for some $s \geq 0$, then a simple count shows that $F_{k+1}\left([0, p-1]^{[m]} \times \mathbb{N}^{[m+1, n-k]}\right)=f_{k}(\Gamma)$ for all $k \leq s$. Hence for this $\Gamma$ a multicomplex $M_{k}=M_{k}(\Gamma)$ of Theorem 3.2 must coincide with the multicomplex $[0, p-1]^{[m]} \times \mathbb{N}^{[m+1, n-k]}$ in degree $k+1$ and all degrees below it.

\section{Monomial sets and Local cohomology}

To complete the proof of Theorem 2.4, and Theorems 1.1 and 3.2 it remains to verify Lemma 2.2. This is the goal of the present section. The proof of the first two parts of the lemma relies on Proposition 2.1 and Eq. (3), and is similar to that of [11, Lemma 6.3], while the proof of the last part utilizes Hochster's theorem [24, Theorem II.4.1], the long exact local cohomology sequence, and the first part of the lemma.

Throughout this section let $\Gamma$ be a $(d-1)$-dimensional simplicial complex $\Gamma \subset\{0,1\}^{[n]}$ and let $u \in G L_{n}(\mathbf{k})$ be a matrix that satisfies the strong Kind-Kleinschmidt condition w.r.t. $\Gamma$. Denote by $\Gamma^{\prime}:=\operatorname{Skel}_{d-2}(\Gamma)$ the $(d-2)$-dimensional skeleton of $\Gamma$. Recall that $B_{u, \Gamma}=\mathbb{N}^{[n]}-\operatorname{in}\left(u I_{\Gamma}\right)$ and $B_{u, \Gamma}\langle k\rangle=B_{u, \Gamma} \cap \mathbb{N}^{[n-k]},-1 \leq k \leq n-1$. To simplify the notation we write $B=B_{u, \Gamma}$ and $B^{\prime}=B_{u, \Gamma^{\prime}}$.

Several observations are in order.

1. Since $u \in G L_{n}(\mathbf{k})$ satisfies the strong Kind-Kleinschmidt condition w.r.t. $\Gamma$, it follows from Eq. (3) that $B\langle d\rangle_{d+1}=\emptyset$ and $B^{\prime}\langle d-1\rangle_{d}=\emptyset$. Therefore,

$$
B^{\prime} \cap B\langle d-1\rangle_{d} \subseteq B^{\prime} \cap \mathbb{N}_{d}^{[n-d+1]}=B^{\prime}\langle d-1\rangle_{d}=\emptyset,
$$

and so

$$
B\langle d-1\rangle_{d} \subseteq B-B^{\prime} .
$$

2. The following is an easily verifiable decomposition of $\mathbb{N}^{[n]}$ (see Figure 1):

$$
\mathbb{N}^{[n]}=\bigcup_{k=0}^{n} \bigcup_{\mu \in \mathbb{N}_{k}^{[n-k+1]}} \mu \mathbb{N}^{[n-k+1, n]} .
$$

Since $B$ is a multicomplex, $B \cap \mu \mathbb{N}^{[n-k+1, n]} \neq \emptyset$ if and only if $\mu \in B$. Thus $B\langle d\rangle_{d+1}=$ $\emptyset$ together with Eq. (7) implies that $B \subseteq \dot{\bigcup}_{k=0}^{d} \dot{\bigcup}_{\mu \in B\langle k-1\rangle_{k}} \mu \mathbb{N}^{[n-k+1, n]}$.

We are now ready to prove the first two parts of Lemma 2.2 asserting that $\left|B\langle k-1\rangle_{k}\right|=$ $f_{k-1}(\Gamma)$ for $k \geq 0$ and that $\mu \mathbb{N}^{[n-k+1, n]} \subseteq B$ for all $\mu \in B\langle k-1\rangle_{k}$, or equivalently (by the above remark) that $\left|B\langle k-1\rangle_{k}\right|=f_{k-1}(\Gamma), k \geq 0$, and

$$
B=\bigcup_{k=0}^{d} \bigcup_{\mu \in B\langle k-1\rangle_{k}} \mu \mathbb{N}^{[n-k+1, n]} .
$$



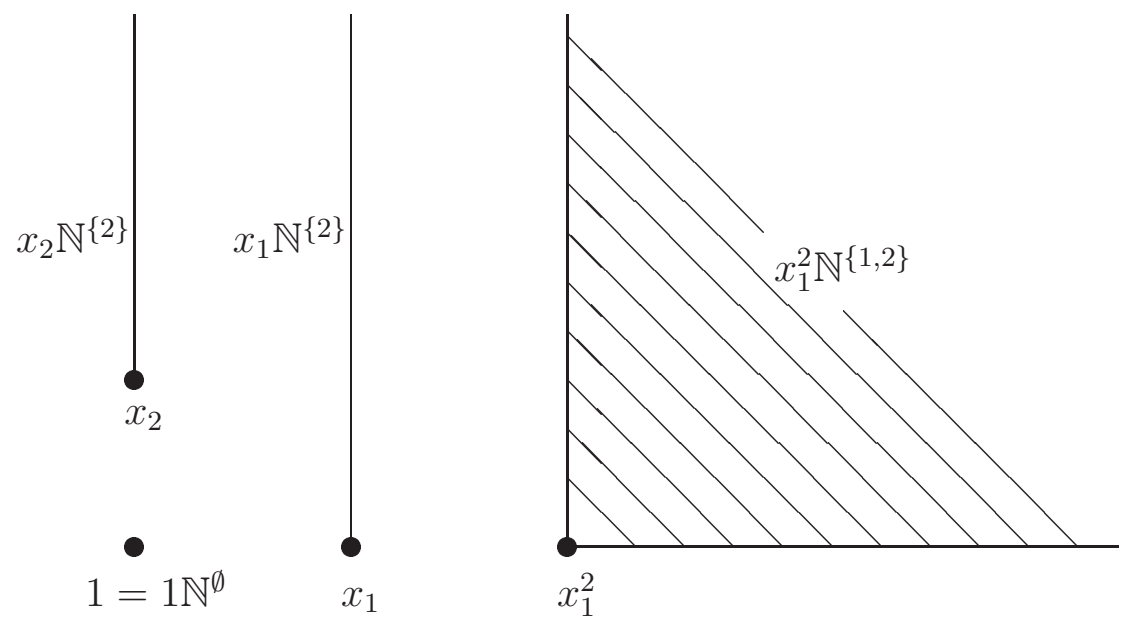

Figure 1: Decomposition of $\mathbb{N}^{\{1,2\}}$

Proof: We apply induction on $d$. If $d=0$, then either $\Gamma=\{1\}$ or $\Gamma=\emptyset$, and so either $I_{\Gamma}=\left\langle x_{1}, \ldots, x_{n}\right\rangle$ or $I_{\Gamma}=\langle 1\rangle$. In the former case $B\langle-1\rangle=B_{0}=\{1\}=1 \mathbb{N}^{\emptyset}$, while in the latter case $B\langle-1\rangle=B_{0}=\emptyset$, and the statement clearly holds.

Assume now that $d>0$ and that $\Gamma^{\prime}=\operatorname{Skel}_{d-2}(\Gamma) \subset \Gamma$ satisfies the assertion, that is, $\left|B^{\prime}\langle k-1\rangle_{k}\right|=f_{k-1}\left(\Gamma^{\prime}\right)$ for $k \geq 0$ and $B^{\prime}=\bigcup_{k=0}^{d-1} \bigcup_{\mu \in B^{\prime}\langle k-1\rangle_{k}} \mu \mathbb{N}^{[n-k+1, n]}$. Since the ideals $I_{\Gamma}$ and $I_{\Gamma^{\prime}}$ coincide up to degree $d-1$, it follows that $B_{k}=B_{k}^{\prime}$ for all $k \leq d-1$, and so $B\langle k-1\rangle_{k}=B^{\prime}\langle k-1\rangle_{k}$ for all $k \leq d-1$. Thus

$$
\begin{gathered}
\left|B\langle k-1\rangle_{k}\right|=\left|B^{\prime}\langle k-1\rangle_{k}\right|=f_{k-1}\left(\Gamma^{\prime}\right)=f_{k-1}(\Gamma) \quad \text { for all } k \leq d-1, \text { and } \\
B \subseteq \bigcup_{k=0}^{d} \bigcup_{\mu \in B\langle k-1\rangle_{k}} \mu \mathbb{N}^{[n-k+1, n]}=B^{\prime} \dot{\cup}\left(\bigcup_{\mu \in B\langle d-1\rangle_{d}} \mu \mathbb{N}^{[n-d+1, n]}\right) .
\end{gathered}
$$

Hence we have

$$
B-B^{\prime} \subseteq \bigcup_{\mu \in B\langle d-1\rangle_{d}} \mu \mathbb{N}^{[n-d+1, n]}
$$

Restricting Eq. (9) to monomials of degree $d$ and comparing it with Eq. (6), we infer that $B\langle d-1\rangle_{d}=B_{d}-B_{d}^{\prime}$. Since $P\left(B-B^{\prime}, t\right)=\frac{f_{d-1}(\Gamma) t^{d}}{(1-t)^{d}}$ (by Proposition 2.1(1,3)), it follows that

$$
\left|B\langle d-1\rangle_{d}\right|=\left|B_{d}-B_{d}^{\prime}\right|=f_{d-1}(\Gamma) .
$$

Finally, since for every monomial $\mu, P\left(\mu \mathbb{N}^{[n-d+1, n]}, t\right)=\frac{t^{\operatorname{deg} \mu}}{(1-t)^{d}}$, we obtain that the generating function of $\dot{\bigcup}_{\mu \in B\langle d-1\rangle_{d}} \mu \mathbb{N}^{[n-d+1, n]}$ equals $\frac{\left|B\langle d-1\rangle_{d}\right| t^{d}}{(1-t)^{d}}=\frac{f_{d-1}(\Gamma) t^{d}}{(1-t)^{d}}$, that is, it coincides with $P\left(B-B^{\prime}, t\right)$. The latter fact implies that the inclusion in Eq. (9) is in fact equality. This establishes Eq. (8).

We now turn to the proof of the last part of Lemma 2.2. This will require the following facts and definitions. If $N$ is a $\mathbf{k}[\mathbf{x}]$-module and $I \subseteq \mathbf{k}[\mathbf{x}]$ is an ideal, then

$$
\left(0:_{N} I\right):=\{\mu \in N \mid \mu I=0\} \text { and }\left(0:_{N} I^{\infty}\right):=\left\{\mu \in N \mid \mu I^{r}=0 \text { for some } r \geq 1\right\} .
$$


If $N=\mathbf{k}[\mathbf{x}]$, we write $(0: I)$ instead of $(0: \mathbf{k}[\mathbf{x}] I)$. Also if $I=\langle f\rangle$, it is customary to write $(0: f)$ and $\left(0: f^{\infty}\right)$ instead of $(0:\langle f\rangle)$ and $\left(0:\langle f\rangle^{\infty}\right)$, respectively. For a $\mathbf{k}[\mathbf{x}]$-module $N$, denote by $H^{i}(N)$ the $i$ th local cohomology of $N$ with respect to the irrelevant maximal ideal $\mathbf{m}=\left\langle x_{1}, \ldots, x_{n}\right\rangle$ of $\mathbf{k}[\mathbf{x}]$ (see e.g. [24, Def. I.6.1]). Recall that $H^{i}(N)$ is graded whenever $N$ is, and that

$$
H^{0}(N)=\left\{\mu \in N: \mu \mathbf{m}^{r}=0 \text { for some } r \geq 1\right\}=\left(0:_{N} \mathbf{m}^{\infty}\right) .
$$

If $N$ is a graded $\mathbf{k}[\mathbf{x}]$-module, write $N_{j}$ to denote its $j$ th homogeneous component. (Thus $N_{j}$ is a k-vector space.)

As in Section 2, consider the family $J\langle k\rangle=u I_{\Gamma}+\left\langle x_{n-k+1}, \ldots, x_{n}\right\rangle, 0 \leq k \leq n$, of graded $\mathbf{k}[\mathbf{x}]$-ideals, and define the corresponding family $N\langle k\rangle:=\mathbf{k}[\mathbf{x}] / J\langle k\rangle, 0 \leq k \leq n$, of graded $\mathbf{k}[\mathbf{x}]$-modules. Recall that

$$
Z\langle k\rangle=Z_{u, \Gamma}\langle k\rangle=\left\{\nu \in B\langle k\rangle: \nu x_{n-k} \notin B\right\}, 0 \leq k \leq n-1 .
$$

Lemma 2.2(3) asserting that $\left|Z\langle k\rangle_{k}\right|=\beta_{k-1}(\Gamma)$ and $Z\langle k\rangle_{l}=\emptyset$ for $l \geq k+1$ is then an immediate corollary of the following two claims.

Lemma $4.1\left|Z\langle k\rangle_{k}\right|=\operatorname{dim}_{\mathbf{k}} H^{0}(N\langle k\rangle)_{k}$ and $Z\langle k\rangle_{l}=\emptyset$ for all $0 \leq k \leq n-1$ and $l \geq k+1$.

Lemma 4.2 For all $i \geq 0$ and $0 \leq s \leq n$,

$$
H^{i}(N\langle s\rangle)_{k} \cong \begin{cases}0 & \text { if } k>s \\ \widetilde{H}_{i+k-1}(\Gamma) & \text { if } k=s\end{cases}
$$

In particular, $\operatorname{dim}_{\mathbf{k}} H^{0}(N\langle k\rangle)_{k}=\beta_{k-1}(\Gamma)$.

Proof of Lemma 4.1: The exact sequence

$$
0 \longrightarrow\left(0:_{N\langle k\rangle} x_{n-k}\right) \longrightarrow N\langle k\rangle=\mathbf{k}[\mathbf{x}] / J\langle k\rangle \longrightarrow \mathbf{k}[\mathbf{x}] /\left(J\langle k\rangle: x_{n-k}\right) \longrightarrow 0
$$

and Proposition 2.1(2) imply that

$$
\begin{aligned}
\operatorname{dim}_{\mathbf{k}}\left(0:_{N\langle k\rangle} x_{n-k}\right)_{l} & =\operatorname{dim}_{\mathbf{k}}(\mathbf{k}[\mathbf{x}] / J\langle k\rangle)_{l}-\operatorname{dim}_{\mathbf{k}}\left(\mathbf{k}[\mathbf{x}] /\left(J\langle k\rangle: x_{n-k}\right)\right)_{l} \\
& =\left|B\langle k\rangle_{l}\right|-\left|B\langle k\rangle_{l}-Z\langle k\rangle_{l}\right|=\left|Z\langle k\rangle_{l}\right| \quad \text { for all } l \geq 0 .
\end{aligned}
$$

If $l \geq k+1$, then the fact that $Z\langle k\rangle_{l}=\emptyset$ is immediate from Eq. (8). Thus

$$
\left(0:_{N\langle k\rangle} x_{n-k}\right)_{l}=\{0\} \quad \text { for all } l \geq k+1,
$$

and so $\mu \mathbf{m} \neq 0$ for some $\mu \in(N\langle k\rangle)_{k}$ implies, by Eq. (11), that $(\mu \mathbf{m}) x_{n-k}^{r} \neq 0$ for all $r \geq 1$, and hence that $\mu \mathbf{m}^{r+1} \neq 0$. Therefore,

$$
H^{0}(N\langle k\rangle)_{k}=\left(0:_{N\langle k\rangle} \mathbf{m}^{\infty}\right)_{k}=\left(0:_{N\langle k\rangle} \mathbf{m}\right)_{k} .
$$


Similarly, if $\nu \in(N\langle k\rangle)_{k}$ and $i \leq n-k$ are such that $N\langle k\rangle_{k+1} \ni x_{i} \nu \neq 0$, then by Eq. (11), $\left(x_{i} \nu\right) x_{n-k} \neq 0$, and hence $\nu x_{n-k} \neq 0$. Therefore,

$$
\left(0:_{N\langle k\rangle} \mathbf{m}\right)_{k}=\left(0:_{N\langle k\rangle} x_{n-k}\right)_{k},
$$

which together with $\operatorname{dim}_{\mathbf{k}}\left(0:_{N\langle k\rangle} x_{n-k}\right)_{k}=|Z\langle k\rangle|_{k}$ (see Eq. (10)) establishes the lemma.

Proof of Lemma 4.2: The proof is by induction on $s$. If $s=0$ then $N\langle 0\rangle=\mathbf{k}[x] / u I_{\Gamma}$ is isomorphic (as a graded algebra) to the Stanley-Reisner ring of $\Gamma$, and the assertion follows from Hochster's theorem [24, Theorem II.4.1]. For $s>0$, define $L^{s}:=N\langle s\rangle /\left(0:_{N\langle s\rangle} x_{n-s}\right)$ and consider the short exact sequence

$$
0 \longrightarrow L^{s}(-1) \stackrel{\cdot x_{n-s}}{\longrightarrow} N\langle s\rangle \longrightarrow N\langle s+1\rangle \longrightarrow 0,
$$

where $L^{s}(-1)$ denotes $L^{s}$ with the grading shifted by 1 . It gives rise to the long exact local cohomology sequence

$$
\cdots \longrightarrow H^{i}(N\langle s\rangle) \longrightarrow H^{i}(N\langle s+1\rangle) \longrightarrow H^{i+1}\left(L^{s}(-1)\right) \longrightarrow H^{i+1}(N\langle s\rangle) \longrightarrow \cdots
$$

Let $k \geq s+1$. Then by the induction hypothesis $H^{i}(N\langle s\rangle)_{k}=H^{i+1}(N\langle s\rangle)_{k}=0$, and thus the above sequence yields

$$
H^{i}(N\langle s+1\rangle)_{k} \cong H^{i+1}\left(L^{s}\right)_{k-1} \cong H^{i+1}(N\langle s\rangle)_{k-1} \cong \begin{cases}0 & \text { if } k>s+1 \\ \widetilde{H}_{i+k-1}(\Gamma) & \text { if } k=s+1\end{cases}
$$

Here the last step follows from the induction hypothesis, while the penultimate step follows from the fact that $\left(0:_{N\langle s\rangle} x_{n-s}\right)$ is a module of finite length (i.e., it is finite-dimensional over k) - see Eq. (11), and so all local cohomology modules (except the 0th one) of $N\langle s\rangle$ coincide with those of $L^{s}=N\langle s\rangle /\left(0:_{N\langle s\rangle} x_{n-s}\right)$.

We conclude this section with several remarks.

Remark 4.3 Since, as follows from Eq. (11), $\left(0:_{N\langle k\rangle} x_{n-k}\right)$ is a module of finite length for all $0 \leq k \leq n-1$, the sequence $x_{n}, x_{n-1}, \ldots, x_{1}$ is an almost regular $N\langle 0\rangle$-sequence in the sense of [1]. The fact that $\left|Z\langle k\rangle_{k}\right|=\operatorname{dim}_{\mathbf{k}}\left(0:_{N\langle k\rangle} x_{n-k}\right)_{k}$ (see Eq. (10)) combined with [1, Cor. 1.2] and with Hochster's formula [10] on the algebraic Betti numbers of the Stanley-Reisner ideal can thus be used to provide another proof of Lemma 2.2(3). Moreover equations (10) and (11) together with [1, Thm. 1.1 and Cor. 1.2] imply that the ideals $I_{\Gamma}$ and $\operatorname{in}\left(u I_{\Gamma}\right)$ have the same extremal Betti numbers whenever $u$ satisfies the strong Kind-Kleinschmidt condition with respect to $\Gamma$, a result previously known only for generic $u$ ([3], [1]).

Remark 4.4 Define the squarefree operation $\Phi: \bigcup_{k=0}^{n} \mathbb{N}_{k}^{[n-k+1]} \rightarrow\{0,1\}^{[n]}$ by

$$
\Phi\left(x_{i_{1}} x_{i_{2}} \cdots x_{i_{k}}\right):=x_{i_{1}} x_{i_{2}+1} \cdots x_{i_{k}+k-1}, \quad \text { where } i_{1} \leq i_{2} \leq \cdots \leq i_{k} .
$$


For a simplicial complex $\Gamma \subseteq\{0,1\}^{n}$ and a matrix $u \in G L_{n}(\mathbf{k})$ let

$$
\Delta_{u, \Gamma}:=\bigcup_{k=0}^{n} \Phi\left(B_{u, \Gamma}\langle k-1\rangle_{k}\right) \subseteq\{0,1\}^{[n]} .
$$

If $u$ is generic and $\mathbf{k}$ is a field of characteristic zero then the set $\Delta(\Gamma):=\Delta_{u, \Gamma}$ is a (shifted) simplicial complex [11]. This complex was introduced in [11] where it was called the algebraic shifting of $\Gamma$. For a nongeneric $u$ that satisfies the strong Kind-Kleinschmidt condition w.r.t. $\Gamma, \Delta_{u, \Gamma}$ is just a "simplicial set" that (by Lemma 2.2(2)) has the same " $f$-numbers" as $\Gamma$. It would be interesting to determine which subsets of $\{0,1\}{ }^{[n]}$ can be realized as $\Delta_{u, \Gamma}$ for some $\Gamma$ and $u$.

\section{$5 \quad$ Shifting colored complexes}

In this section we extend some of the above results, most notably Lemma $2.2(1,2)$, to polynomial rings (simplicial complexes) with $\mathbb{N}^{r}$-grading. We then introduce the notion of colored algebraic shifting and discuss some of its properties. To this end, we assume that the set of variables $V$ of the polynomial ring $\mathbf{k}[V]$ is endowed with an ordered partition $\left(V_{1}, \ldots, V_{r}\right)$ (i.e., a sequence of nonempty and pairwise disjoint subsets of $V$ whose union is $V)$. We write $V_{j}=\left\{x_{j, 1}, \cdots, x_{j, n_{j}}\right\}$ where $n_{j}=\left|V_{j}\right|$ and $1 \leq j \leq r$, and we identify the set of monomials of $\mathbf{k}[V]$ with $\mathbb{N}^{\left[n_{1}\right]} \times \cdots \times \mathbb{N}^{\left[n_{r}\right]}$ and the set of squarefree monomials of $\mathbf{k}[V]$ with $\{0,1\}^{\left[n_{1}\right]} \times \cdots \times\{0,1\}^{\left[n_{r}\right]}$ via the ring isomorphism $\mathbf{k}[V] \cong \mathbf{k}\left[x_{1}, \ldots, x_{n_{1}}\right] \otimes_{\mathbf{k}} \cdots \otimes_{\mathbf{k}} \mathbf{k}\left[x_{1}, \ldots, x_{n_{r}}\right]$.

Let $\mathbf{e}_{j} \in \mathbb{N}^{r}, 1 \leq j \leq r$, denote the $j$ th unit coordinate vector in $\mathbb{N}^{r}$, i.e., $\mathbf{e}_{j}=$ $\left(\delta_{1 j}, \ldots, \delta_{r j}\right)$. Define an $\mathbb{N}^{r}$-grading of $\mathbf{k}[V]$ by setting $\operatorname{deg} x=\mathbf{e}_{j}$ if $x \in V_{j}, 1 \leq j \leq r$. If $M \subseteq \mathbf{k}[V]$ is a multicomplex and $\mathbf{b} \in \mathbb{N}^{r}$, we denote by $f_{\mathbf{b}}(M)$ the number of monomials in $M$ whose $\mathbb{N}^{r}$-degree is $\mathbf{b}$. The flag $f$-vector of $M$ is the vector $\left(f_{\mathbf{b}}(M): \mathbf{b} \in \mathbb{N}^{r}\right)$. It is a refinement of the usual $F$-vector, since $F_{i}(M)=\sum f_{\mathbf{b}}(M)$ where the sum is over all $\mathbf{b} \in \mathbb{N}^{r}$ such that $\sum \mathbf{b}_{j}=i$ (hence for a simplicial complex $\Gamma, f_{i-1}(\Gamma)=\sum\left\{f_{\mathbf{b}}(\Gamma): \mathbf{b} \in\right.$ $\left.\left.\mathbb{N}^{r}, \sum \mathbf{b}_{j}=i\right\}\right)$.

Let $G:=G L_{n_{1}}(\mathbf{k}) \times \cdots \times G L_{n_{r}}(\mathbf{k})$. Every matrix $u=\left(u^{1}, \cdots, u^{r}\right) \in G$ defines an $\mathbb{N}^{r}$-graded automorphism of $\mathbf{k}[V]$ via $u\left(x_{j, l}\right)=\sum_{i=1}^{n_{j}} u_{i l}^{j} x_{j, i}$. In particular, if $\Gamma \subseteq$ $\{0,1\}^{\left[n_{1}\right]} \times \cdots \times\{0,1\}^{\left[n_{r}\right]}$ is a simplicial complex, then $u I_{\Gamma}$ is a homogeneous ideal (w.r.t $\mathbb{N}^{r}$-grading).

Consider an arbitrary linear order $\succ$ on $V=\cup_{j=1}^{r} V_{j}$ whose restriction to $V_{j}$ (for every $1 \leq j \leq r)$ is given by $x_{j, 1} \succ x_{j, 2} \succ \cdots \succ x_{j, n_{j}}$. In contrast with the $r=1$ case, many such orders exist if $r>1$. As in Section 2, we define

$$
B=B_{u, \Gamma}^{\succ}:=\mathbb{N}^{\left[n_{1}\right]} \times \cdots \times \mathbb{N}^{\left[n_{r}\right]}-\operatorname{in}_{\succ}\left(u I_{\Gamma}\right) \quad \text { and } \quad B_{\mathbf{c}}:=\{\mu \in B: \operatorname{deg} \mu=\mathbf{c}\},
$$

where $\operatorname{in}_{\succ}\left(u I_{\Gamma}\right)$ is the reverse lexicographic initial ideal of $u I_{\Gamma}$ w.r.t. order $\succ$ on $V, u \in$ $G$, and $\mathbf{c} \in \mathbb{N}^{r}$. Thus $B_{u, \Gamma}^{\succ}$ is a multicomplex that provides a $\mathbf{k}$-basis for $\mathbf{k}[V] / u I_{\Gamma}$. Since $\mathbf{k}[V] / u I_{\Gamma}$ and $\mathbf{k}[V] / I_{\Gamma}$ are isomorphic $\mathbb{N}^{r}$-graded algebras, [21, Eq. (4)] implies the 
following refinement of Proposition 2.1(3). (For $\mathbf{t}=\left(t_{1}, \ldots, t_{r}\right)$ and $\mathbf{c}=\left(c_{1}, \cdots, c_{r}\right) \in \mathbb{N}^{r}$, write $\mathbf{t}^{\mathbf{c}}=t_{1}^{c_{1}} \cdots t_{r}^{c_{r}}$.)

Proposition 5.1 The $\mathbb{N}^{r}$-graded generating function of $B=B_{u, \Gamma}^{\succ}$, $P(B, \mathbf{t}):=\sum_{\mathbf{c} \in \mathbb{N}^{r}}\left|B_{\mathbf{c}}\right| \mathbf{t}^{\mathbf{c}}$, equals $\sum_{\mathbf{c} \in \mathbb{N}^{r}} \frac{f_{\mathbf{c}}(\Gamma) \mathbf{t}^{\mathbf{c}}}{\left(1-t_{1}\right)^{c_{1} \ldots\left(1-t_{r}\right)^{c_{r}}}}$

We are now in a position to provide an extension of Lemma 2.2(1,2) to $\mathbb{N}^{r}$-grading. We start with the following observation. For a simplicial complex $\Gamma$, let $\Gamma_{j}(1 \leq j \leq r)$ be the induced subcomplex of $\Gamma$ on the vertex set $V_{j}$. If $u=\left(u^{1}, \cdots, u^{r}\right) \in G$ then $\left(u I_{\Gamma}\right)_{l \mathbf{e}_{j}} \cong \mathbf{k} \otimes \cdots \otimes \mathbf{k} \otimes\left(u^{j} I_{\Gamma_{j}}\right)_{l} \otimes \mathbf{k} \cdots \otimes \mathbf{k}$ for all $l \in \mathbb{N}$ and $1 \leq j \leq r$, and so $\left(B_{u, \Gamma}^{\succ}\right)_{l \mathbf{e}_{j}}=\{1\} \times \cdots \times\left(B_{u^{j}, \Gamma_{j}}\right)_{l} \times \cdots \times\{1\}$. Since $B_{u, \Gamma}^{\succ}$ is a multicomplex, this yields

$$
B_{u, \Gamma}^{\succ} \subseteq B_{u^{1}, \Gamma_{1}} \times \cdots \times B_{u^{r}, \Gamma_{r}} .
$$

We say that a matrix $u=\left(u^{1}, \cdots, u^{r}\right) \in G$ satisfies the strong colored Kind-Kleinschmidt condition with respect to $\Gamma$ if for all $1 \leq j \leq r, u^{j}$ satisfies the strong Kind-Kleinschmidt condition with respect to $\Gamma_{j}$ (as defined in Section 2). In analogy with Section 2 we also define

$$
B_{u, \Gamma}^{\succ}\langle\mathbf{c}\rangle:=B_{u, \Gamma}^{\succ} \cap\left(\mathbb{N}^{\left[n-c_{1}\right]} \times \cdots \times \mathbb{N}^{\left[n-c_{r}\right]}\right), \quad \text { where } \mathbf{c}=\left(c_{1}, \ldots, c_{r}\right) \in \mathbb{Z}^{r}, c_{i} \leq n_{i} .
$$

Write $\mathbf{e}=\mathbf{e}_{1}+\cdots+\mathbf{e}_{r}$. Lemma 2.2(1,2) has the following multigraded version.

Lemma 5.2 Let $\Gamma$ be a simplicial complex on $V$ and let $u \in G$ be a matrix satisfying the strong colored Kind-Kleinschmidt condition with respect to $\Gamma$. Then for all $\mathbf{c}=\left(c_{1}, \ldots, c_{r}\right) \in \mathbb{N}^{r}$ such that $0 \leq c_{j} \leq n_{j}, 1 \leq j \leq r$, the following holds

1. $\mu \cdot\left(\mathbb{N}^{\left[n_{1}-c_{1}+1, n_{1}\right]} \times \cdots \times \mathbb{N}^{\left[n_{r}-c_{r}+1, n_{r}\right]}\right) \subseteq B_{u, \Gamma}^{\succ}$ for all $\mu \in B_{u, \Gamma}^{\succ}\langle\mathbf{c}-\mathbf{e}\rangle_{\mathbf{c}}$.

2. $\left|B_{u, \Gamma}^{\succ}\langle\mathbf{c}-\mathbf{e}\rangle_{\mathbf{c}}\right|=f_{\mathbf{c}}(\Gamma)$.

Proof: Since $u^{j}$ satisfies the strong Kind-Kleinschmidt condition with respect to $\Gamma_{j}$ $(1 \leq j \leq r)$ we have by Eq. (8) that

$$
B_{u^{j}, \Gamma_{j}}=\bigcup_{c_{j}=0}^{\operatorname{dim} \Gamma_{j}+1} \bigcup_{\mu_{j} \in B_{u j, \Gamma_{j}}\left\langle c_{j}-1\right\rangle_{c_{j}}} \mu_{j} \mathbb{N}^{\left[n_{j}-c_{j}+1, n_{j}\right]}
$$

which together with Eq. (12) and the fact that $B_{u, \Gamma}^{\succ}$ is a multicomplex implies that

$$
B_{u, \Gamma}^{\succ} \subseteq \bigcup_{\mathbf{c} \in \mathbb{N}^{r}, c_{j} \leq \operatorname{dim} \Gamma_{j}+1} \bigcup_{\mu \in B_{u, \Gamma}^{\succ}\langle\mathbf{c}-\mathbf{e}\rangle_{\mathbf{c}}} \mu \cdot\left(\mathbb{N}^{\left[n_{1}-c_{1}+1, n_{1}\right]} \times \cdots \times \mathbb{N}^{\left[n_{r}-c_{r}+1, n_{r}\right]}\right) .
$$

Thus to prove the lemma it suffices to show that the inclusion in the above equation is in fact equality and that $\left|B_{u, \Gamma}^{\succ}\langle\mathbf{c}-\mathbf{e}\rangle_{\mathbf{c}}\right|=f_{\mathbf{c}}(\Gamma)$. We verify this claim by induction on $r$ and on $\sum_{j=1}^{r} \operatorname{dim} \Gamma_{j}$. The case of $r=1$ is covered by the proof of Lemma 2.2(1,2), while the case of $\operatorname{dim} \Gamma_{j}=-1$ for some $1 \leq j \leq r$ is equivalent to having only $r-1$ sets $V_{i}$. 
Hence for the inductive step assume that $\operatorname{dim} \Gamma_{j} \geq 0,1 \leq j \leq r$, and that the assertion holds for all complexes $\Gamma_{1} * \cdots * \Gamma_{j}^{\prime} * \cdots * \Gamma_{r}, 1 \leq j \leq r$, where $\Gamma_{j}^{\prime}$ is the codimension one skeleton of $\Gamma_{j}$, and $*$ denotes the simplicial join (as defined in Example 3.1). The rest of the proof is completely analogous to that of Lemma 2.2(1,2): replace $B_{u, \Gamma}-B_{u, \Gamma^{\prime}}$ with $B_{u, \Gamma}^{\succ}-\bigcup_{j=1}^{r} B_{u, \Gamma_{1} * \cdots * \Gamma_{j}^{\prime} * \cdots * \Gamma_{r}}^{\succ}$ and use Proposition 5.1 instead of Proposition 2.1(3). We omit the details.

We now come to the central definition of this section - the notion of colored algebraic shifting. As in the case of the classical algebraic shifting it involves generic initial ideals. In particular we need the following fact.

Theorem 5.3 Let $I \subseteq \mathbf{k}[V]$ be an $\mathbb{N}^{r}$-graded ideal. There is a Zariski open set $U=U(I)$ in $G$ and an ideal $J$ such that $\operatorname{in}_{\succ}(u I)=J$ for all $u \in U$.

Theorem 5.3 is a multigraded analog of [8, Theorem 15.18]. We omit its verification, which is a straightforward generalization of the proof of [8, Theorem 15.18]. The ideal $J$ defined in the theorem is called the G-generic initial ideal of $I$, and a matrix $u \in U(I)$ is called a G-generic matrix w.r.t. I.

We say that a monomial ideal $I \subseteq \mathbf{k}[V]$ is strongly color-stable if it satisfies the following condition: for every monomial $\mu \in I$ and for every $1 \leq j \leq r$, if $\mu$ is divisible by $x_{j, i}$ and $1 \leq l<i$, then $\mu x_{j, l} / x_{j, i} \in I$. [8, Theorem 15.20] combined with [8, Theorem 15.23] has the following multigraded version. (We do not supply its proof here, since the proof of $[8$, Theorem 15.20$]$ carries over almost verbatim to the $\mathbb{N}^{r}$-graded case.)

Theorem 5.4 If $\mathbf{k}$ is a field of characteristic zero, then the G-generic initial ideal of an $\mathbb{N}^{r}$-graded ideal of $\mathbf{k}[V]$ is strongly color-stable.

The squarefree map $\Phi$ from Remark 4.4 gives rise to the color-squarefree map

$$
\tilde{\Phi}: \bigcup_{\mathbf{c} \in \mathbb{N}^{r}, c_{j} \leq n_{j}} \mathbb{N}_{c_{1}}^{\left[n-c_{1}+1\right]} \times \cdots \times \mathbb{N}_{c_{r}}^{\left[n-c_{r}+1\right]} \rightarrow\{0,1\}^{\left[n_{1}\right]} \cdots \times \cdots\{0,1\}^{\left[n_{r}\right]}
$$

defined by $\left(\mu_{1}, \cdots, \mu_{r}\right) \mapsto\left(\Phi\left(\mu_{1}\right), \cdots, \Phi\left(\mu_{r}\right)\right)$. This is a one-to-one map that preserves $\mathbb{N}^{r}$-grading. In analogy with the classical algebraic shifting (see Remark 4.4 ) we make the following definition.

Definition 5.5 Let $\Gamma \subseteq\{0,1\}^{\left[n_{1}\right]} \times \cdots \times\{0,1\}^{\left[n_{r}\right]}$ be a simplicial complex, and let $u \in$ $U\left(I_{\Gamma}\right)$ be a $G$-generic matrix. The set

$$
\tilde{\Delta}_{\succ}(\Gamma):=\bigcup_{\mathbf{c} \in \mathbb{N}^{r}, c_{j} \leq n_{j}} \tilde{\Phi}\left(B_{u, \Gamma}^{\succ}\langle\mathbf{c}-\mathbf{e}\rangle_{\mathbf{c}}\right) \subseteq\{0,1\}^{\left[n_{1}\right]} \times \cdots \times\{0,1\}^{\left[n_{r}\right]}
$$

is called the colored algebraic shifting of $\Gamma$ (induced by $\succ$ ). 
Performing colored algebraic shifting amounts to (i) computing the $G$-generic ideal of the Stanley-Reisner ideal; (ii) considering the set of all monomials that do not lie in the resulting ideal; and (iii) applying the color-squarefree operation $\tilde{\Phi}$ to all monomials in the above set on which it is well-defined. The reason for the name "colored shifting" is part 1 of the following theorem.

Theorem 5.6 If $\mathbf{k}$ is a field of characteristic zero, then the colored algebraic shifting $\tilde{\Delta}_{\succ}(\Gamma)$ of a simplicial complex $\Gamma$ is a simplicial complex. Moreover,

1. $\tilde{\Delta}_{\succ}(\Gamma)$ is color-shifted - for every $\mu \in \tilde{\Delta}_{\succ}(\Gamma)$ and every $1 \leq j \leq r$, if $\mu$ is divisible by $x_{j, i}$ but is not divisible by $x_{j, l}$ where $i<l \leq n_{j}$, then $\mu x_{j, l} / x_{j, i} \in \tilde{\Delta}_{\succ}(\Gamma)$;

2. $\tilde{\Delta}_{\succ}(\Gamma)$ and $\Gamma$ have the same flag $f$-vector: $f_{\mathbf{c}}\left(\tilde{\Delta}_{\succ}(\Gamma)\right)=f_{\mathbf{c}}(\Gamma)$ for all $\mathbf{c} \in \mathbb{N}^{r}$.

Proof: It is a routine exercise to derive the fact that $\tilde{\Delta}_{\succ}(\Gamma)$ is closed under divisibility, and hence is a simplicial complex, as well as the fact that it is color-shifted from Theorem 5.4 and Definition 5.5 (cf. [2, Lemma 1.2]). Since the strong colored Kind-Kleinschmidt condition w.r.t $\Gamma$ is an open condition, there exists a $G$-generic w.r.t $I_{\Gamma}$ matrix that satisfies this condition. The equality $f_{\mathbf{c}}\left(\tilde{\Delta}_{\succ}(\Gamma)\right)=f_{\mathbf{c}}(\Gamma)$ for all $\mathbf{c} \in \mathbb{N}^{r}$ then follows from Lemma 5.2(2) and Definition 5.5.

In analogy with shifted complexes, color-shifted complexes have a very simple combinatorial structure (see Theorems 5.7 and 6.2 below). At the same time (again similarly to the usual algebraic shifting), colored algebraic shifting preserves several combinatorial, algebraic and topological properties of colored simplicial complexes such as the flag $f$-numbers (Theorem 5.6(2)) and the property of being Cohen-Macaulay (at least when applied to a-balanced complexes and for a particular choice of $\succ$, see Theorem 6.5). Because of these features we expect colored algebraic shifting to become a useful tool in the study of face numbers. We however do not know at present whether $\beta\left(\tilde{\Delta}_{\succ}(\Gamma)\right)=\beta(\Gamma)$ even for a completely balanced (that is, e-balanced) $\Gamma$. The desire to establish this fact is partially explained by the following theorem.

Theorem 5.7 Let $\Gamma$ be a color-shifted complex such that $f_{\mathbf{e}}(\Gamma) \neq 0$. Then for an arbitrary field $\mathbf{k}$ and for all $i \geq 0$

$$
\beta_{i-1}(\Gamma)=\mid\left\{\mu \in \max (\Gamma): \operatorname{deg}_{\mathbb{N}}(\mu)=i \text { and } \mu \text { is not divisible by } x_{j, n_{j}} \forall 1 \leq j \leq r\right\} \mid,
$$

where $\max (\Gamma)$ denotes the set of the facets of $\Gamma$.

This theorem is a colored analog of [6, Theorem 4.3]. The proof uses the notions of the link and the star of a face $\nu$ in $\Gamma$ :

$$
\begin{aligned}
& \operatorname{lk}_{\Gamma}(\nu):=\{\mu \in \Gamma: \operatorname{gcd}(\mu, \nu)=1 \text { and } \mu \cdot \nu \in \Gamma\}, \\
& \operatorname{st}_{\Gamma}(\nu):=\left\{\nu^{\prime} \cdot \mu: \nu^{\prime} \mid \nu \text { and } \mu \in \operatorname{lk}_{\Gamma}(\nu)\right\} .
\end{aligned}
$$

$\operatorname{st}_{\Gamma}(\nu)$ is a cone over the simplex $\nu$, and hence is a contractible complex as long as $1 \neq \nu$. In the following, $\|\Gamma\|$ denotes the geometric realization of $\Gamma$. 
Proof: Consider the subcomplex $\Sigma:=\cup_{j=1}^{r} \mathrm{st}_{\Gamma}\left(x_{j, n_{j}}\right)$ of $\Gamma$. Since $f_{\mathbf{e}}(\Gamma)>0$, and since $\Gamma$ is color-shifted, we infer that the monomial $\prod_{j=1}^{r} x_{j, n_{j}}$ and all its divisors are faces of $\Gamma$. Thus for any $\emptyset \neq L \subseteq[r]$, the subcomplex $\cap_{l \in L} \operatorname{st}_{\Gamma}\left(x_{l, n_{l}}\right)=\operatorname{st}_{\Gamma}\left(\prod_{l \in L} x_{l, n_{l}}\right)$ is nonempty and contractible, and so by the Nerve Theorem (see e.g. $[4,(10.6)]$ ), $\Sigma$ is contractible. Therefore the projection map $\|\Gamma\| \rightarrow\|\Gamma\| /\|\Sigma\|$ is a homotopy equivalence (see [4, (10.2)]). Now, $\mu \in \Gamma-\Sigma$ if and only if $\mu \in \Gamma$, but $x_{j, n_{j}} \cdot \mu \notin \Gamma$ for all $1 \leq j \leq r$ which (by colorshiftedness of $\Gamma$ ) happens if and only if $x \cdot \mu \notin \Gamma$ for all $x \in V$. This implies that all the elements of $\Gamma-\Sigma$ are facets:

$$
\Gamma-\Sigma=\left\{\mu \in \max (\Gamma): \mu \text { is not divisible by } x_{j, n_{j}} \forall 1 \leq j \leq r\right\}
$$

and so the contraction of $\|\Sigma\|$ to a point turns each $(i-1)$-dimensional face of $\Gamma-\Sigma$ into an $(i-1)$-dimensional sphere. Hence $\|\Gamma\| /\|\Sigma\|$ is a wedge of spheres (wedged at the image of $\|\Sigma\|$ ), each sphere corresponding to an element of $\Gamma-\Sigma$, and the assertion follows.

\section{Cohen-Macaulay and Buchsbaum balanced com- plexes}

The goal of this section is to provide a simpler proof of the Stanley-Björner-Frankl theorem $([21,5])$ that characterizes all possible flag $f$-vectors of a-balanced Cohen-Macaulay complexes, and then to generalize the necessity part of this result to certain conditions on the face numbers and Betti numbers of a-balanced Buchsbaum complexes.

Historically, a simplicial complex $\Gamma$ is called Cohen-Macaulay over $\mathbf{k}$ (CM, for short) if its Stanley-Reisner ring $\mathbf{k}[\mathbf{x}] / I_{\Gamma}$ is Cohen-Macaulay, and $\Gamma$ is called Buchsbaum (over $\mathbf{k})$ if $\mathbf{k}[\mathbf{x}] / I_{\Gamma}$ is Buchsbaum. Here we adopt the following topological characterizations of CM and Buchsbaum complexes due to Reisner [18] and Schenzel [19], respectively, as their definitions. (Recall that a simplicial complex is pure if all its facets are of the same dimension.)

Definition 6.1 A $(d-1)$-dimensional simplicial complex $\Gamma$ is Cohen-Macaulay (over $\mathbf{k}$ ) if $\widetilde{H}_{i-1}\left(\operatorname{lk}_{\Gamma}(\mu)\right)=0$ for every face $\mu \in \Gamma$ including $\mu=1$ and all $i<d-\operatorname{deg}(\mu) . \Gamma$ is Buchsbaum (over $\mathbf{k}$ ) if it is pure and the link of every vertex is Cohen-Macaulay (over $\mathbf{k}$ ).

It follows easily from the above definition that every CM complex is pure. Although the converse is false in general, it does hold for color-shifted complexes. (Recall that $M$ is an a-colored multicomplex for some $\mathbf{a}=\left(a_{1}, \cdots, a_{r}\right) \in \mathbb{Z}_{+}^{r}$, if $f_{\mathbf{b}}(M)=0$ unless $\mathbf{b} \in \mathbb{N}^{r}$ is coordinate-wise $\leq \mathbf{a}$. A simplicial complex $\Gamma$ is $\mathbf{a}$-balanced if it is a-colored and $\operatorname{dim} \Gamma+1=\sum_{i=1}^{r} a_{i}$.)

Theorem 6.2 Let $\Gamma$ be an a-balanced color-shifted complex. Then $\Gamma$ is Cohen-Macaulay (over any field) if and only if $\Gamma$ is pure. 
Proof: We show by induction on $\operatorname{dim}(\Gamma)$ that if $\Gamma$ is pure then it is $\mathrm{CM}$. The base case $\operatorname{dim} \Gamma=0$ holds, since all 0-dimensional complexes are CM. Write $\mathbf{a} / \mathbf{e}_{i}$ to denote the vector $\mathbf{a}-\mathbf{e}_{i}$ if $a_{i}>1$, and the vector $\left(a_{1}, \cdots, a_{i-1}, a_{i+1}, \cdots, a_{r}\right)$ otherwise. Also write $V / x_{i, l}$ to denote $V-\left\{x_{i, l}\right\}$ if $a_{i}>1$, and $V-V_{i}$ otherwise. Since $\Gamma$ is a-balanced and pure, the complex $\operatorname{lk}_{\Gamma}\left(x_{i, l}\right)$ is $\mathbf{a} / \mathbf{e}_{i}$-balanced and pure for all $1 \leq i \leq r$ and $1 \leq l \leq n_{i}$. Moreover, since $\Gamma$ is color-shifted, $\operatorname{lk}_{\Gamma}\left(x_{i, l}\right)$ is color-shifted as a complex on the vertex set $V / x_{i, l}$. Thus by the induction hypothesis, $\mathrm{lk}_{\Gamma}(x)$ is $\mathrm{CM}$ for every vertex $x \in \Gamma$. Finally, since $\Gamma$ is pure, $\Gamma$ has no facets of dimension $<\operatorname{dim}(\Gamma)$, and so by Theorem 5.7 all Betti numbers of $\Gamma$ vanish except possibly for the top-dimensional one, yielding that $\Gamma$ is $\mathrm{CM}$. (The condition $f_{\mathbf{e}}(\Gamma)>0$ of Theorem 5.7 is satisfied since $\Gamma$ is a-balanced, and so even $f_{\mathbf{a}}(\Gamma)>0$.)

Let $\Gamma$ be an a-balanced complex. As in [21, p. 146], define the flag h-numbers of $\Gamma$, $h_{\mathbf{c}}(\Gamma), \mathbf{c} \in \mathbb{N}^{r}$, by the following relation

$$
\frac{\sum_{\mathbf{c} \in \mathbb{N}^{r}} h_{\mathbf{c}} \mathbf{t}^{\mathbf{c}}}{\left(1-t_{1}\right)^{a_{1}} \cdots\left(1-t_{r}\right)^{a_{r}}}=\sum_{\mathbf{c} \in \mathbb{N}^{r}} \frac{f_{\mathbf{c}}(\Gamma) \mathbf{t}^{\mathbf{c}}}{\left(1-t_{1}\right)^{c_{1}} \cdots\left(1-t_{r}\right)^{c_{r}}} .
$$

Equivalently, $h_{\mathbf{c}}(\Gamma)=\sum_{\mathbf{b} \in \mathbb{N}^{r}, b_{i} \leq c_{i}} f_{\mathbf{b}}(\Gamma) \prod_{i=1}^{r}(-1)^{c_{i}-b_{i}}\left(\begin{array}{l}a_{i}-b_{i} \\ c_{i}-b_{i}\end{array}\right)$ and

$$
f_{\mathbf{c}}(\Gamma)=\sum_{\mathbf{b} \in \mathbb{N}^{r}, b_{i} \leq c_{i}} h_{\mathbf{b}}(\Gamma) \prod_{i=1}^{r}\left(\begin{array}{c}
a_{i}-b_{i} \\
c_{i}-b_{i}
\end{array}\right), \quad \mathbf{c} \in \mathbb{N}^{r} .
$$

Thus $h_{\mathbf{c}}(\Gamma)=0$ unless $\mathbf{c}$ is coordinate-wise $\leq \mathbf{a}$. The vector $\left(h_{\mathbf{c}}(\Gamma): \mathbf{c} \in \mathbb{N}^{r}\right)$ is called the flag $h$-vector. It is a refinement of the $h$-vector: $h_{i}(\Gamma)=\sum_{\mathbf{c}} h_{\mathbf{c}}(\Gamma)$ for all $0 \leq i \leq$ $\operatorname{dim}(\Gamma)+1$, where the sum is over all $\mathbf{c} \in \mathbb{N}^{r}$ with $\sum_{j=1}^{r} c_{j}=i$.

We now provide a simple proof of the Stanley-Björner-Frankl theorem $[21,5]$. We set $W:=\cup_{j=1}^{r}\left\{x_{j, n_{j}-a_{j}+1}, \ldots, x_{j, n_{j}}\right\} \subset V$, and $G^{\prime}:=G L_{n_{1}-a_{1}}(\mathbf{k}) \times \cdots \times G L_{n_{r}-a_{r}}(\mathbf{k})$.

Theorem 6.3 A sequence $h=\left\{h_{\mathbf{b}}: \mathbf{b} \in \mathbb{N}^{r}, b_{i} \leq a_{i}(i=1, \ldots, r)\right\}$ is the flag $h$-vector of an a-balanced CM complex on the vertex set $V$ if and only if it is the flag $f$-vector of an a-colored multicomplex on the set of variables $V-W$.

Proof of sufficiency: Let $h$ be the flag $f$-vector of an a-colored multicomplex $M$ on the set of variables $V-W$, and let $\mathbf{k}$ be a field of characteristic zero. Define $I_{M}$ to be the $\mathbf{k}$-span of all monomials in $\mathbf{k}[V-W]$ that do not lie in $M$. Since $M$ is a multicomplex, $I_{M}$ is a monomial ideal. Let $J_{M}$ be the $G^{\prime}$-generic initial ideal of $I_{M}$, and let $B_{M}$ be the set of monomials of $\mathbf{k}[V-W]$ that do not lie in $J_{M}$. Define the following subsets of monomials of $\mathbf{k}[V]$

$$
B\langle\mathbf{c}-\mathbf{e}\rangle:=\cup_{\mu \in B_{M}} \mu \cdot\left(\mathbb{N}^{\left[n_{1}-a_{1}+1, n_{1}-c_{1}+1\right]} \times \cdots \times \mathbb{N}^{\left[n_{r}-a_{r}+1, n_{r}-c_{r}+1\right]}\right), \mathbf{c} \in \mathbb{N}^{r}, c_{i} \leq a_{i}
$$

and the following a-colored set of squarefree monomials

$$
\Gamma:=\cup_{\mathbf{c} \in \mathbb{N}^{r}, c_{i} \leq a_{i}} \tilde{\Phi}\left(B\langle\mathbf{c}-\mathbf{e}\rangle_{\mathbf{c}}\right) .
$$


We claim that $\Gamma$ is an a-balanced simplicial CM complex whose flag $h$-vector is $h$. Since $J_{M}$ is strongly color-stable (Theorem 5.4), it follows easily from the definition of $\tilde{\Phi}$ that $\Gamma$ is a simplicial complex and that it is color-shifted.

To show that $\Gamma$ is a-balanced and CM, it suffices (by Theorem 6.2) to verify that $\Gamma$ is pure of dimension $\left(\sum a_{i}\right)-1$. And indeed, let $\mu \in \Gamma$ be of degree $\mathbf{c}$, where, say, $c_{i_{0}}<a_{i_{0}}$. To see that $\mu$ is not a facet, consider $\tilde{\mu}:=\tilde{\Phi}^{-1}(\mu) \in B\langle\mathbf{c}-\mathbf{e}\rangle_{\mathbf{c}}$ and denote by $k \in \mathbb{N}$ the maximal integer such that $x_{i_{0}, n_{i_{0}}-c_{i_{0}}+1}^{k}$ divides $\tilde{\mu}$. Then (since $c_{i_{0}}<a_{i_{0}}$ and from the definition of $B\langle\mathbf{c}-\mathbf{e}\rangle)$,

$$
\tilde{\nu}:=\tilde{\mu} x_{i_{0}, n_{i_{0}}-c_{i_{0}}}^{k+1} / x_{i_{0}, n_{i_{0}}-c_{i_{0}}+1}^{k}
$$

is an element of $B\left\langle\mathbf{c}+\mathbf{e}_{i_{0}}-\mathbf{e}\right\rangle_{\mathbf{c}+\mathbf{e}_{i_{0}}}$, and so $\tilde{\Phi}(\tilde{\nu}) \in \Gamma$. The assertion follows since applying $\tilde{\Phi}$ to both sides of (14) yields $\tilde{\Phi}(\tilde{\nu})=\mu x_{i_{0}, n_{i_{0}}-k}$.

Finally, since passing to $G^{\prime}$-generic initial ideals preserves the $\mathbb{N}^{r}$-graded Hilbert series, $P\left(B_{M}, \mathbf{t}\right)=P(M, t)=\sum h_{\mathbf{b}} \mathbf{t}^{\mathbf{b}}$, and so the definition of $B\langle\mathbf{c}-\mathbf{e}\rangle$ implies that

$$
P(B\langle\mathbf{c}-\mathbf{e}\rangle, \mathbf{t})=\frac{\sum h_{\mathbf{b}} \mathbf{t}^{\mathbf{b}}}{\prod_{i=1}^{r}\left(1-t_{i}\right)^{a_{i}-c_{i}+1}} .
$$

Hence $f_{\mathbf{c}}(\Gamma)=\left|B\langle\mathbf{c}-\mathbf{e}\rangle_{\mathbf{c}}\right|=\sum h_{\mathbf{b}} \prod_{i=1}^{r}(-1)^{c_{i}-b_{i}}\left(\begin{array}{c}-\left(a_{i}-c_{i}+1\right) \\ c_{i}-b_{i}\end{array}\right)=\sum h_{\mathbf{b}} \prod_{i=1}^{r}\left(\begin{array}{c}a_{i}-b_{i} \\ c_{i}-b_{i}\end{array}\right)$, and we infer from Eq. (13) that $h_{\mathbf{b}}(\Gamma)=h_{\mathbf{b}}$ for all $\mathbf{b} \in \mathbb{N}^{r}$.

For the rest of this section we consider only a-balanced complexes on $V=\dot{\cup}_{j=1}^{r} V_{j}$, $|V|=n$. We set $d:=\sum_{j=1}^{r} a_{j}$ and we put the following interlacing restriction on the ordering $\succ$ of $V$ : we require that the $d$-element subset $W=\cup_{j=1}^{r}\left\{x_{j, n_{j}-a_{j}+1}, \ldots, x_{j, n_{j}}\right\}$ form the tail segment of $V$ w.r.t. $\succ$. We identify the $i$-th element of $V$ in the $\succ$-order with $x_{i}(1 \leq i \leq n)$, and we use this identification to embed the matrix group $G$ into $G L_{n}(\mathbf{k})$. Thus $W$ is identified with $\left\{x_{n-d+1}, \ldots, x_{n}\right\}$, and so $B_{u, \Gamma}^{\succ}\langle\mathbf{a}\rangle=B_{u, \Gamma}\langle d\rangle$. An immediate but useful observation is that under this identification, a matrix $u=\left(u^{1}, \cdots, u^{r}\right) \in G \subset$ $G L_{n}(\mathbf{k})$ that satisfies the strong colored Kind-Kleinschmidt condition w.r.t. a-balanced $\Gamma$ also satisfies the usual noncolored Kind-Kleinschmidt condition w.r.t $\Gamma$, and hence that for such $u$, the elements of $W$ provide a homogeneous (w.r.t $\mathbb{N}^{r}$-grading) system of parameters for $\mathbf{k}[\mathbf{x}] / u I_{\Gamma}$.

Stanley [21, Eq. (7)] showed that the flag $h$-numbers of an a-balanced CM complex $\Gamma$ are equal to dimensions of certain vector spaces. In view of Proposition 2.1(2) his result can be restated as follows.

Lemma 6.4 Let $\Gamma$ be an a-balanced $C M$ complex. If $u \in G \subset G L_{n}(\mathbf{k})$ satisfies the Kind-Kleinschmidt condition w.r.t $\Gamma$, then $\left|B_{u, \Gamma}^{\succ}\langle\mathbf{a}\rangle_{\mathbf{b}}\right|=h_{\mathbf{b}}(\Gamma)$ for all $\mathbf{b} \in \mathbb{N}^{r}$.

The proof of the necessity of conditions of Theorem 6.3 is now immediate:

Proof of necessity: Assume that $\Gamma$ is an a-balanced CM complex on $V$. Let $u \in G$ be a matrix satisfying the strong colored Kind-Kleinschmidt property w.r.t. $\Gamma$. By Lemma 6.4, the set $B_{u, \Gamma}^{\succ}\langle\mathbf{a}\rangle$ is the required a-colored multicomplex on $V-W$.

We close our discussion of a-balanced CM complexes with the following result. 
Theorem 6.5 If $\Gamma$ is an a-balanced CM complex, then its colored algebraic shifting, $\tilde{\Delta}_{\succ}(\Gamma)$, (computed over a field of characteristic zero) is a CM complex as well.

Proof: Since $\tilde{\Delta}_{\succ}(\Gamma)$ is a color-shifted a-balanced complex (Theorem 5.6), to prove that it is CM it suffices (by Theorem 6.2) to check that it is pure. And indeed, if $u \in G$ satisfies the strong colored Kind-Kleinschmidt condition w.r.t. $\Gamma$, then

$$
B_{u, \Gamma}^{\succ}=\bigcup_{\mu \in B_{u, \Gamma}^{\succ}\langle\mathbf{a}\rangle} \mu\left(\mathbb{N}^{\left[n_{1}-a_{1}+1, n_{1}\right]} \times \cdots \times \mathbb{N}^{\left[n_{r}-a_{r}+1, n_{r}\right]}\right),
$$

and the purity of $\tilde{\Delta}_{\succ}(\Gamma)$ follows exactly as in the proof of the sufficiency part of Theorem 6.3.

Eq. (15) is immediate from the usual definition of $\mathrm{CM}$ complexes via regular sequences. An easy way to verify it from the results stated above is to notice that (i) since $B_{u, \Gamma}^{\succ}$ is a multicomplex, the left-hand-side of (15) is contained in its right-hand-side, and (ii) that by Lemma 6.4 and Proposition 5.1 both sides of (15) have the same generating function, namely $\left(\sum_{\mathbf{b} \in \mathbb{N}^{r}} h_{\mathbf{b}} \mathbf{t}^{\mathbf{b}}\right) / \prod\left(1-t_{i}\right)^{a_{i}}$.

The necessity portion of Theorem 6.3 has the following generalization to the face numbers and Betti numbers of a-balanced Buchsbaum complexes. For a $(d-1)$-dimensional Buchsbaum complex $\Gamma$ define

$$
h_{j}^{\prime}(\Gamma):=h_{j}(\Gamma)+\left(\begin{array}{l}
d \\
j
\end{array}\right) \sum_{i=0}^{j-1}(-1)^{j-i-1} \beta_{i-1}(\Gamma) \text { for } j=0,1, \ldots, d .
$$

Thus $h_{0}^{\prime}(\Gamma)=1, h_{1}^{\prime}(\Gamma)=f_{0}(\Gamma)-d$, and if $\Gamma$ is $\mathrm{CM}$ then $h_{j}^{\prime}(\Gamma)=h_{j}(\Gamma)$ for all $j$.

Theorem 6.6 If $\Gamma$ is an a-balanced Buchsbaum complex on $V, d=\sum a_{i}$, and $1 \leq k \leq$ $d-1$ then there exists an a-colored multicomplex $M_{k}$ on $V-W$ such that $F_{k+1}\left(M_{k}\right)=$ $h_{k+1}^{\prime}(\Gamma)$ and $F_{k}\left(M_{k}\right)=h_{k}^{\prime}(\Gamma)-\left(\begin{array}{c}d-1 \\ k\end{array}\right) \beta_{k-1}(\Gamma)$.

A special case of Theorem 6.6 for $\mathbf{a} \in \mathbb{N}^{1}$ was verified in [16] (with an additional restriction that $\mathbf{k}$ be of characteristic zero) and in [17] (for an arbitrary field $\mathbf{k}$ ).

Proof: Let $u=\left(u^{1}, \cdots, u^{r}\right) \in G \subset G L_{n}(\mathbf{k})$ be a matrix satisfying the strong colored Kind-Kleinschmidt condition w.r.t $\Gamma$. Define $M_{k}$ to be the subset of $B_{u, \Gamma}^{\succ}\langle\mathbf{a}\rangle=B_{u, \Gamma}\langle d\rangle$ consisting of all elements of $B_{u, \Gamma}\langle d\rangle_{k+1}$, all elements of $B_{u, \Gamma}\langle d\rangle_{k}-Z_{u, \Gamma}\langle d-1\rangle_{k}$, and all divisors of the latter. (Recall definition of $Z\langle d-1\rangle$ from Section 2.) Since $u$ satisfies the Kind-Kleinschmidt condition w.r.t. $\Gamma$, it follows from [17, Lemma 3.6(b,c)] that $Z_{u, \Gamma}\langle d-$ $1\rangle \subset B_{u, \Gamma}\langle d\rangle$, and that no divisor of $B_{u, \Gamma}\langle d\rangle$ is an element of $Z_{u, \Gamma}\langle d-1\rangle$, and thus that $M_{k}$ is a multicomplex. Also by [17, Lemma 3.6(a)], $\left|B_{u, \Gamma}\langle d\rangle_{j}\right|=h_{j}^{\prime}(\Gamma)$ for all $j \geq 0$, and $Z_{u, \Gamma}\langle d-1\rangle_{k}=\left(\begin{array}{c}d-1 \\ j\end{array}\right) \beta_{k-1}(\Gamma)$, implying the assertion on the $F$-numbers of $M_{k}$. Finally, since $u^{i}$ satisfies the strong Kind-Kleinschmidt condition w.r.t. $\left(a_{i}-1\right)$-dimensional complex $\Gamma_{i}(1 \leq i \leq r), B_{u^{i}, \Gamma_{i}}\left\langle a_{i}\right\rangle_{a_{i}+1}=\emptyset$ by Eq. (3). Eq. (12) then yields that $M_{k} \subseteq B_{u, \Gamma}^{\succ}\langle\mathbf{a}\rangle$ is a-colored. 
There exists a purely numerical (similar in spirit to the Kruskal-Katona theorem) characterization of the $F$-numbers of e-colored multicomplexes due to Frankl, Füredi and Kalai [9, Thm. 1.2]. Hence for the $\mathbf{a}=\mathbf{e}$ case one can easily verify whether two given integer sequences $\left\{h_{j}^{\prime}: 0 \leq j \leq d\right\}$ and $\left\{\beta_{j}: 0 \leq j \leq d-1\right\}$ satisfy the condition of Theorem 6.6. However no numerical characterization of the $F$-numbers of a-colored multicomplexes is known for other values of $\mathbf{a} \in \mathbb{N}^{r}(r>1)$.

\section{References}

[1] A. Aramova and J. Herzog. Almost regular sequences and Betti numbers. American J. Math., 122:689-719, 2000.

[2] A. Aramova, J. Herzog, and T. Hibi. Shifting operations and graded Betti numbers. J. Algebraic Combin., 12:207-222, 2000.

[3] D. Bayer, H. Charalambous, and S. Popescu. Extremal Betti numbers and applications to monomial ideals. J. Algebra, 221 (2):497-512, 1999.

[4] A. Björner. Topological methods. In M. Grötschel R. L. Graham and L. Lovász, editors, Handbook of combinatorics, volume 2, pages 1819-1872. Elsevier, Amsterdam, 1995.

[5] A. Björner, P. Frankl, and R. Stanley. The number of faces of balanced CohenMacaulay complexes and a generalized Macaulay theorem. Combinatorica, 7:23-34, 1987.

[6] A. Björner and G. Kalai. An extended Euler-Poincaré theorem. Acta Math., 161:279303, 1988.

[7] G. Clements and B. Lindström. A generalization of a combinatorial theorem of Macaulay. J. Combin. Theory, 7:230-238, 1969.

[8] D. Eisenbud. Commutative Algebra with a View Towards Algebraic Geometry. Springer-Verlag (Graduate Texts in Mathematics), New York, 1995.

[9] P. Frankl, Z. Furedi, and G. Kalai. The size of the shadow of colored complexes. Math. Scand., 63:169-178, 1988.

[10] M. Hochster. Cohen-Macaulay rings, combinatorics, and simplicial complexes. In B.R. McDonald and R. Morris, editors, Ring Theory II (Proc. Second Oklahoma Conference), pages 171-223. Dekker, New-York, 1977.

[11] G. Kalai. The diameter of graphs of convex polytopes and f-vector theory. In P. Gritzmann and B. Sturmfels, editors, Applied Geometry and Discrete Mathematics - The Victor Klee Festschrift, volume 4 of DIMACS Series in Discrete Mathematics and Theoretical Computer Science, pages 387-411. American Mathematical Society, Providence, RI, 1991. 
[12] J. O. H. Katona. A theorem of finite sets. In Theory of graphs (Proc. Tihany Conf., 1966, P. Erdös and G. Katona, eds.), pages 187-207. Academic Press, New York, and Akadémia Kiadó, Budapest, 1968.

[13] B. Kind and P. Kleinschmidt. Schälbare Cohen-Macaulay-Komplexe und ihre Parametrisierung. Math. Z., 167:173-179, 1979.

[14] J. B. Kruskal. The number of simplices in a complex. In R. Bellman, editor, Mathematical Optimization Techniques, pages 251-278. Univ. of California Press, Berkeley - Los Angeles, 1963.

[15] F. S. Macaulay. Some properties of enumeration in the theory of modular systems. Proc. London Math. Soc., 26:531-555, 1927.

[16] I. Novik. Upper bound theorems for homology manifolds. Israel J. Math., 108:45-82, 1998.

[17] I. Novik. On face numbers of manifolds with symmetry. Advances in Math., 192:183208, 2005.

[18] G. A. Reisner. Cohen-Macaulay quotiens of polynomial rings. Advances in Math., 21:30-49, 1976.

[19] P. Schenzel. On the number of faces of simplicial complexes and the purity of Frobenius. Math. Z., 178:125-142, 1981.

[20] R. Stanley. Cohen-Macaulay complexes. In M. Aigner, editor, Higher Combinatorics, pages 51-62. Reidel, Dordrecht/Boston, 1977.

[21] R. Stanley. Balanced Cohen-Macaulay complexes. Trans. Amer. Math. Soc., 249:139157, 1979.

[22] R. Stanley. On the number of faces of centrally-symmetric simplicial polytopes. Graphs and Combinatorics, 3:55-66, 1987.

[23] R. Stanley. A monotonicity property of $h$-vectors and $h^{*}$-vectors. European J. Combinatorics, 14:251-258, 1993.

[24] R. Stanley. Combinatorics and Commutative Algebra. Birkhäuser, Boston Inc., Boston, MA, 1996. second edition. 